\title{
Non-homogenous analysis of rogue wave probability evolution over a shoal
}

\author{
Saulo Mendes, ${ }^{1,2, *}$ Alberto Scotti, ${ }^{3, \dagger}$ Maura Brunetti, ${ }^{1,2, \ddagger}$ and Jerome Kasparian ${ }^{1,2, \S}$ \\ ${ }^{1}$ Group of Applied Physics, University of Geneva, \\ Chemin de Pinchat 22, 1211 Geneva 4, Switzerland \\ ${ }^{2}$ Institute for Environmental Sciences, University of Geneva, \\ Boulevard Carl-Vogt 66, 1211 Geneva, Switzerland \\ ${ }^{3}$ Department of Marine Sciences, University of North Carolina at Chapel Hill, Chapel Hill, NC, 27599 USA
}

\begin{abstract}
Non-equilibrium evolution of wave fields, as occurring over sudden bathymetry variations, can produce rogue seas with anomalous wave statistics. We handle this process by modifying the Rayleigh distribution through the energetics of second-order theory and a non-homogenous reformulation of the Khintchine theorem. The resulting probability model reproduces the enhanced tail of the probability distribution of unidirectional wave tank experiments. It also describes why the peak of rogue wave probability appears atop the shoal, and explains the influence of depth on variations in peak intensity for different depths. Furthermore, we interpret rogue wave likelihoods in finite depth through the $H-\sigma$ diagram, allowing a quick prediction for the effects of rapid depth change apart from the probability distribution.
\end{abstract}

\section{INTRODUCTION}

Ocean statistics offers numerous applications, particularly in marine and offshore safety [1]. Models for short-term and long-term statistics of water waves are used to define the operating envelope for ocean vessels and fixed offshore structures, respectively. Furthermore, understanding the mechanisms of formation of rogue waves has received a considerable amount of attention in past decades $[2,3]$. Defined as waves at least twice as tall as the significant wave height $[2,4]$, rogue waves present a looming danger to offshore operations $[5,6]$. Theories of rogue wave formation include the Benjamin and Feir instability [7] arising in surface gravity waves, described by the nonlinear Schrödinger equation [3, 8], and linear mechanisms such as constructive interference [9-11]. From a statistical point of view, any particular theory relies on its ability to reproduce the tail of the wave amplitude probability distribution.

Longuet-Higgins [12] applied methods and ideas from signal processing [13] to oceanography (see St Denis and Pierson [14] for a review). In particular, his approach took Rice [13] underlying assumptions of homogeneity and ergodicity for granted. Therefore, the resulting non-dimensional Rayleigh distribution of wave heights cannot account for the varying sea state parameters, such as steepness [15] or depth [16]. The same limitation applies to higher-order analytical distributions $[17,18]$. Though these standard approaches have reached considerable success in explaining the observed directional spectrum and wave properties [19-21], the need for the ergodicity and spatial homogeneity assumptions essentially prevents the use of spectral analysis techniques in unsteady conditions or during isolated events, such as rogue waves [22]. Furthermore, Haver and Andersen [23] were the first to suggest a link between rogue waves and non-stationarity. Nevertheless, we still lack a framework to account for unsteady conditions, such as shoaling. For a review of the consequences of non-stationarity/homogeneity, see appendix A 2.

Following Trulsen et al. [24] laboratory experiments, considerable attention has been given to the shoaling effect on rogue wave formation. Waves approaching a sudden change in bathymetry provide an ideal configuration to probe out-of-equilibrium conditions [25]. Additional experiments [26-31] and numerical studies [32-36] have attested heavier tails than expected by Longuet-Higgins [12]. Nonlinearities and abrupt depth change lead waves out-of-equilibrium, deviating from Gaussian statistics. For a step, these bathymetry effects have been described by nonlinear evolution of interacting free modes with a truncation of the $\mathrm{KdV}$ equation [37,38] and as travelling wavepackets subject to second-order effects in steepness $[39,40]$. However, wave height probability distributions able to describe the laboratory results of Trulsen et al. [31] are still lacking. In fact, Li et al. [41] have obtained a wave crest probability distribution for the step case based on Tayfun [42], but as it is stated in the section following eqs. 36-38 of Tayfun [42], this framework can not lead to non-Gaussian wave height distributions. The present work seeks a probability distribution for non-homogenous conditions encountered by waves undergoing rapid depth change. More precisely, we show that regardless of the pre-shoal probability distribution shape, the transformation of the sea surface elevation and spatial energy density through leading second-order effects in steepness will amplify the rogue wave probability. This amplification depends on the dimensionless depth $\left(k_{p} h\right)$ and significant steepness $(\varepsilon)$.

\footnotetext{
* saulo.dasilvamendes@unige.ch

$\dagger$ ascotti@unc.edu

¥ maura.brunetti@unige.ch

$\S$ jerome.kasparian@unige.ch
} 


\section{BACKGROUND AND GENERAL FORMULATION}

Following Massel [43], we focus on wave and crest heights normalised by the significant wave height $H_{1 / 3}$, respectively, as:

$$
\alpha \equiv \frac{H}{H_{1 / 3}}=\frac{\mathcal{Z}_{c}+\mathcal{Z}_{t}}{H_{1 / 3}} \quad, \quad \beta \equiv \frac{\mathcal{Z}_{c}}{H_{1 / 3}}
$$

where $H=\mathcal{Z}_{c}+\mathcal{Z}_{t}$ is the crest-to-trough height (hereafter denoted as wave height), $\mathcal{Z}_{c}$ is the adjacent crest height and $\mathcal{Z}_{t}$ the trough depth. Assuming we can approximate $H=2 \mathcal{Z}_{c}$ in narrow-banded seas, the Rayleigh exceedance probability distribution reads [12]:

$$
\mathbb{P}_{\mathcal{R}}\left(H>\alpha H_{1 / 3}\right) \equiv \mathcal{R}_{\alpha}=\int_{\alpha}^{+\infty} f_{\alpha_{0}} d \alpha_{0}=\int_{\alpha}^{+\infty} 4 \alpha_{0} e^{-2 \alpha_{0}^{2}} d \alpha_{0}=e^{-2 \alpha^{2}}
$$

with $f_{\alpha}$ denoting the probability density function of wave heights. For broader spectra we define the vertical asymmetry $[44,45]$ between crest and trough as the ratio between crest and wave height [46]. Hence, whenever it becomes necessary to convert normalised crests into normalised wave heights in broad-banded seas, it is computed as follows (see section 6.1 of Mendes et al. [18]):

$$
\mathfrak{S}_{0}(\alpha) \equiv 2\left\langle\frac{\beta}{\alpha}\right\rangle_{r} \approx \frac{2 \eta_{1 / 3}}{1+\eta_{1 / 3}}\left[1+\frac{2 \eta_{1 / 3} \operatorname{Re}(\sqrt{\alpha-1})}{7+2 \operatorname{Re}(\sqrt{\alpha-1})}\right], \eta_{1 / 3} \equiv\left(\frac{\left\langle\mathcal{Z}_{c}\right\rangle_{r}}{\left\langle\mathcal{Z}_{t}\right\rangle_{r}}\right)_{H>H_{1 / 3}},
$$

with $\langle\cdot\rangle_{r}$ denoting a wave record average. Experimental works typically use 20-min records. Remarkably, the vertical asymmetry is related to the skewness $\mu_{3}$ through the approximation $\eta_{1 / 3} \approx 1+\left\langle\mu_{3}\right\rangle_{r}$ (see eq. 14 and figure 8 of Mendes et al. [18]). Moreover, considering the correlation between asymmetry, skewness and significant steepness reported by Guedes Soares et al. [47], the asymmetry is weakly dependent on the bandwidth $\nu$ due to the bound $\mu_{3}\left(1+\nu^{2}\right) \lesssim k H_{1 / 3}[48]$.

\section{A. Equilibrium Wave Statistics}

In view of the equivalence between the spectral analysis of spatial and time domains (see appendix A 1), we start by considering the spatial energy density around $x$ [49], calculated over one spectral zero-crossing wavelength $\bar{\lambda}$ [43]:

$$
\mathcal{E}=\frac{\rho}{2 \bar{\lambda}} \int_{x}^{x+\bar{\lambda}}\left[g(\zeta+h(x))^{2}+\int_{-h(x)}^{\zeta}\left(u_{1}^{2}+u_{3}^{2}\right) d z\right] d x
$$

where $\rho$ is the density, $g$ is the gravitational acceleration, $h$ the water column depth, $x$ the direction of motion and $z$ the vertical axis so that $\vec{g}=-g \hat{z}, \zeta$ is the sea surface elevation and $u_{i}=\partial \Phi / \partial x_{i}$ is the $i$-th velocity component derived from the velocity potential. Indeed, Trulsen et al. [31] experiments had a length of slope of $1.6 \mathrm{~m}$ while the typical peak wavelength ranged from 1 to $1.8 \mathrm{~m}$, thus validating our calculation above. For an irregular wavefield obeying the solution of linear theory [50] with uncorrelated random phases $\theta_{i}$ and amplitudes $a_{i}$, one has the surface elevation [42]:

$$
\zeta_{1}(x, t)=\sum_{i} a_{i} \cos \left(k_{i} x-\omega_{i} t+\theta_{i}\right)
$$

where the $i$-th components have wavenumber $k_{i}$ and frequency $\omega_{i}$. Assuming $\zeta / h \ll 1$ and $\langle\zeta\rangle \approx 0$ for the second integral interval in eq. (4) [49], the energy reads:

$$
\mathcal{E}_{\mathrm{AIRY}}=\frac{1}{8} \rho g H^{2}=\frac{1}{2} \rho g a^{2} \quad \therefore \quad \mathscr{E}_{\mathrm{AIRY}}=\frac{\mathcal{E}_{\mathrm{AIRY}}}{\rho g}=\frac{1}{2} \sum_{i} a_{i}^{2},
$$

where $a$ is the wave train amplitude. Then, using the spatial counterpart of the Khintchine [51] theorem, which relates the spectral density of a spatial series with its autocorrelation in homogenous processes, one concludes (see appendix A 1):

$$
R_{x}(0)=\left\langle\zeta^{2}\right\rangle=\int_{0}^{+\infty} S(k) d k \equiv m_{0}=\mathscr{E}_{A I R Y}
$$


with $S(k)$ denoting the unidirectional ocean energy spectrum [43]. This water wave solution features a Rayleigh distribution of wave heights in the form $\mathbb{P}\left(H>H_{0}\right)=e^{-H_{0}^{2} / 8 m_{0}}$ in an irregular wave field with narrow-banded spectrum [12]. In the next section we shall challenge the assumption of homogeneity implied by the use of the Khintchine [51] theorem, paving the way for the analysis of non-equilibrium wave statistics.

\section{NON-EQUILIBRIUM WAVE STATISTICS}

Despite the usefulness of the Airy [50] formulation for the spectral analysis of water waves, the evolution of the ocean surface is neither stationary, spatially homogenous nor ergodic [52, 53]. Therefore, higher-order (unsteady) corrections to the Khintchine [51] theorem should be considered. In the spirit of Longuet-Higgins [54] and Das and Nason [55], we do so by considering a small correction $\Gamma$ to the aforementioned theorem. During the shoaling we have:

$$
R_{x}(0)=\left\langle\zeta^{2}\right\rangle=\Gamma \int_{0}^{+\infty} S(k) d k=\Gamma m_{0} \quad, \quad|\Gamma-1| \ll 1
$$

This approach assumes that the distribution of phases is not too far from uniform, as in Tayfun [56] who described a maximum 5\% deviation. In fact, the $\Gamma$ correction to the first moment is much smaller than to the second moment, such that the system can still be treated as mean-ergodic (see appendix A 2 and eq. (C7)).

Indeed, for weakly homogenous processes, it is customary to fix a free parameter $A$ such that the area under the spectral curve is equal to the mean power [43], e.g. $R_{x}(0)=\int_{0}^{\infty} S(k) d k$ for a one-sided spectrum. Instead, we choose $A$ such that the area under the spectral curve matches the spatial energy density $(\mathscr{E})$ during homogeneous stages and it reduces to the autocorrelation in the Gaussian case, i.e. in the strictly homogeneous case prior to the shoal. For non-homogenous processes, this is not the case and the methods for finding a spectrum produce anomalies and undesirable features [57-61]. This implies that there is no canonical or unique way to define a non-homogeneous Khintchine theorem [59], such that the ratio $\left\langle\zeta^{2}\right\rangle / \mathscr{E}$ computes the deviation from homogeneity.

Let us for now focus on how the wave statistics will adapt to a non-homogeneous correction parameter $\Gamma$ in the ocean. To first order, the probability density of wave heights must fulfil the narrow-band identity:

$$
\int_{0}^{+\infty} f(H) H^{2} d H=8\left\langle\zeta^{2}\right\rangle
$$

Due to the difficulty to convert surface elevation distributions into crest and height distributions in broad-banded seas [62], eq. (9) replaces the typical envelope approach to find the wave height distribution directly. Therefore, neglecting skewness $\mu_{3}$ and kurtosis $\mu_{4}$, the change in the ratio $\left\langle\zeta^{2}\right\rangle / m_{0} \rightarrow \Gamma \cdot\left(\left\langle\zeta^{2}\right\rangle / m_{0}\right)$ applied to eq. (2) relates the $2 \alpha^{2}$ to $\left\langle\zeta^{2}\right\rangle / m_{0}$, resulting in the narrow-banded correction:

$$
\mathcal{R}_{\alpha, \Gamma}\left(H>\alpha H_{1 / 3}\right)=\int_{\alpha}^{+\infty} \frac{4 \alpha_{0}}{\Gamma} e^{-2 \alpha_{0}^{2} / \Gamma} d \alpha_{0}=e^{-2 \alpha^{2} / \Gamma},
$$

that is, the initial wave train Gaussian statistics will be affected by bathymetry and its exceedance probability $\mathcal{R}_{\alpha}$ will be transformed into $\mathcal{R}_{\alpha, \Gamma}$ by the shoaling process.

Following the same energetics argument as in section II A, let us generalise the velocity potential [63] and surface elevation in eq. (5) to an irregular wave field subject to second-order effects in steepness, with uncorrelated random amplitudes and phases (a brief discussion is provided in appendix A 2):

$$
\begin{aligned}
\Phi(x, z, t) & =\sum_{i} \sum_{m} \frac{\Omega_{m, i}\left(k_{i} h\right)}{m k_{i}} \cosh (m \varphi) \sin (m \phi) \\
\zeta(x, t) & =\sum_{i} \sum_{m} \tilde{\Omega}_{m, i}\left(k_{i} h\right) \cos (m \phi)
\end{aligned}
$$

with the auxiliary variables $\varphi=k_{i}(z+h)$ and $\phi=k_{i}\left(x-c_{m, i} t+\theta_{i}\right)$ where $c_{m, i}=c_{m}\left(k_{i}\right)$ is the phase velocity of the $i$-th spectral component of $m$-th order in steepness. To allow an analytical treatment, the above expression for surface elevation contains no directional effects nor wave-wave interaction and is restricted to the super-harmonic term of the second-order correction. This approximation is supported by Dingemans [63], Forristall [64]. In fact, sub-harmonics are at least one order of magnitude smaller than the super-harmonic (see figure 7 of Li et al. [40]), and given that the super-harmonic contributes only to a $2-3 \%$ change in the energy correction due to shoaling, one should expect the sub-harmonic term to not be fundamental to our analysis. Due to this approximation, however, the treatment 
is not equivalent to Stokes waves, whose propagation in deep water having a narrow-banded spectrum leads to the modulational instability $[2,3,8]$.

Under the framework above, we subtract the fixed depth $h_{0}^{2} / 2$ term from the energy and can prove for a depth variation $\partial h(x) / \partial x \ll 1$ (see appendix B):

$$
\left\langle\zeta^{2}\right\rangle=\frac{1}{2} \sum_{i} \sum_{m} \tilde{\Omega}_{m, i} \quad ; \quad \mathscr{E}=\frac{1}{4} \sum_{i} \sum_{m}\left[\tilde{\Omega}_{m, i}^{2}+\Omega_{m, i}^{2} \cdot \frac{\sinh (2 m k h)}{2 m g k}\right] .
$$

In the limit $i \rightarrow \infty$, we can treat the leading order coefficients $\left(\Omega_{m}, \tilde{\Omega}_{m}\right)$ as being decomposed into a series of even powers of steepness coupled to factored out trigonometric functions $\left(\chi_{m}, \tilde{\chi}_{m}\right)$ (see appendix B), leading to:

$$
\begin{array}{r}
\mathscr{E}=\frac{a^{2}}{4}\left[1+\left(\frac{\pi \varepsilon}{4}\right)^{2} \chi_{1}+\ldots\right]+\sum \frac{a^{2}}{4}\left[1+\left(\frac{\pi \varepsilon}{4}\right)^{2} \tilde{\chi}_{1}+\ldots\right] . \\
\left\langle\zeta^{2}\right\rangle=\frac{a^{2}}{2}\left[1+\left(\frac{\pi \varepsilon}{4}\right)^{2} \tilde{\chi}_{1}+\left(\frac{\pi \varepsilon}{4}\right)^{4} \tilde{\chi}_{2}+\ldots\right] .
\end{array}
$$

Hence, due to the expressions in eq. (8) we get:

$$
\Gamma=\frac{\left\langle\zeta^{2}\right\rangle}{\mathscr{E}}=\frac{1+\sum_{p}\left(\frac{\pi \varepsilon}{4}\right)^{2 p} \tilde{\chi}_{p}}{1+\frac{1}{2} \sum_{p}\left(\frac{\pi \varepsilon}{4}\right)^{2 p}\left(\tilde{\chi}_{p}+\chi_{p}\right)}
$$

This expression demonstrates that the effect of energetics is reduced to the coefficients of eq. (13). We assume that waves before the shoal propagate on a flat bottom and follow the Gaussian distribution associated with the linear wave theory. Afterwards, due to the bathymetry change, second-order corrections become relevant since a much larger steepness is to be taken into account (see Eagleson [65]). Consequently, out-of-equilibrium dynamics will deform an initially Gaussian distribution due to higher-order effects in steepness. In order to apply the same correction to an arbitrary initial distribution, appendix $\mathrm{C}$ shows that modelling any bathymetry change by a transition from first to second-order terms is very effective. The $\Gamma$ correction only depends on $\varepsilon$ and $k h$. Additionally, it is independent on slope of the shoaling, provided the latter is sufficiently steep [35]. Thus, it is applicable to Trulsen et al. [31] experiments that featured a slope of 1/3.8. Indeed, results by Gramstad et al. [33] suggest that the rogue wave probability is an order of magnitude more sensible to a relative change in dimensionless depth $k_{p} h$ than in the slope of the shoal. Recently, Fu et al. [66] has also demonstrated that for steep-shoaling slopes the probability of rogue waves remains the same.

\section{A. Second-Order Statistics}

Under the validity of the above assumptions, the spatial energy density for a second-order perturbation in the narrow-banded case reads (see eq. (B9)):

$$
\mathcal{E}=\frac{1}{2} \rho g a^{2}\left[1+\left(\frac{\pi \varepsilon}{4}\right)^{2}\left(\frac{\tilde{\chi}_{1}+\chi_{1}}{2}\right)\right] \quad, \quad k a=\pi \varepsilon \cdot \mathfrak{S}_{0}
$$

with coefficients reading (see figure 1a):

$$
\tilde{\chi}_{1}=\left[\frac{\cosh k h[2+\cosh (2 k h)]}{\sinh ^{3} k h}\right]^{2}, \chi_{1}=\frac{9 \cosh (2 k h)}{\sinh ^{6} k h} .
$$

This model is valid provided the Ursell number being Ur $=H \lambda^{2} / h^{3}=\varepsilon(2 \pi / k h)^{3} \leqslant 8 \pi^{2} / 3$ [49, 67]. Hence, for small amplitudes $(\zeta \ll h)$ :

$$
\mathscr{E}=\frac{1}{2} \sum_{i} a_{i}^{2}+\frac{\pi^{2}}{16}\left(\tilde{\chi}_{1}+\chi_{1}\right) \sum_{i} \frac{a_{i}^{4}}{\lambda_{i}^{2}} \equiv m_{0}
$$

whereas the surface total variance reads:

$$
\left\langle\zeta^{2}\right\rangle=\frac{1}{2} \sum_{i} a_{i}^{2}+\frac{\tilde{\chi}_{1} \pi^{2}}{8} \sum_{i} \frac{a_{i}^{4}}{\lambda_{i}^{2}} .
$$



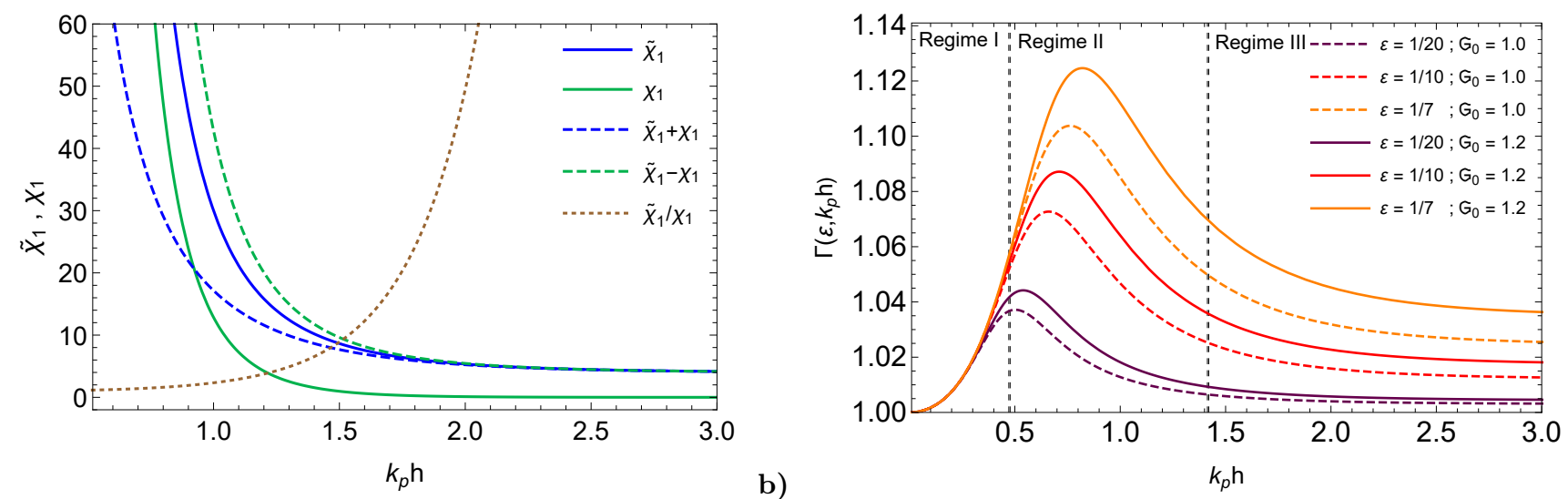

b)

FIG. 1: (a) Trigonometric coefficients $\left(\chi_{m}, \tilde{\chi}_{m}\right)$ of the second-order model and (b) correction parameter $\Gamma$ as a function of steepness $\varepsilon=H_{1 / 3} / \bar{\lambda}$ and dimensionless depth $k_{\mathrm{p}} h$ in both narrow-banded $\left(\mathfrak{S}_{0}=1.0\right)$ and broad-banded seas $\left(\mathfrak{S}_{0}=1.2\right)$.

In order to consider the whole ensemble of waves, we employ the wave number at the peak of the spectrum, $k_{\mathrm{p}}$, in the dimensionless depth $k_{\mathrm{p}} h$, and the zero-crossing wavelength $\bar{\lambda}$ in the significant steepness $\varepsilon=H_{1 / 3} / \bar{\lambda}[43]$. Thus, in the limit of a large number of wave components, the previous expressions read

$$
\frac{2\left\langle\zeta^{2}\right\rangle}{a^{2}}=1+\left(\frac{\pi \varepsilon}{4}\right)^{2} \tilde{\chi}_{1} \quad ; \quad \frac{2 \mathscr{E}}{a^{2}}=1+\left(\frac{\pi \varepsilon}{4}\right)^{2} \frac{\left(\tilde{\chi}_{1}+\chi_{1}\right)}{2} .
$$

The narrow-banded correction for a group of waves over a changing bathymetry is:

$$
\Gamma=\frac{\left\langle\zeta^{2}\right\rangle}{\mathscr{E}}=\frac{32+2 \tilde{\chi}_{1} \pi^{2} \varepsilon^{2}}{32+\left(\tilde{\chi}_{1}+\chi_{1}\right) \pi^{2} \varepsilon^{2}} \approx 1+\left(\frac{\pi \varepsilon}{4}\right)^{2}\left(\frac{\tilde{\chi}_{1}-\chi_{1}}{2}\right) .
$$

$\Gamma$ exceeds 1 for all depths, with a maximum of up to 1.13 in intermediate depths $\left(k_{\mathrm{p}} h \sim 0.5-1\right)$ and an asymptotic behaviour for deep water (see figure 1b). This shape defines three regimes, as marked on figure 1b. In shallow water (Regime I), a shoal reducing the depth will reduce $\Gamma$, hence the exceedance probability. Conversely, beyond the maximum of $\Gamma$ (Regime II), the shoal will increase $\Gamma$ and the exceedance probability. Finally, as long as the shoal stays within the third regime, the depth variation will translate into a negligible change of $\Gamma$, hence will have no consequence on the exceedance probability. Such absence in amplification is similar to the second-order height distribution in [42] in deep water (see section IIIE). This behaviour will allow to simplify investigations of shoals starting in deep water $\left(k_{\mathrm{p}} h \gtrsim 2\right)$, well within Regime III. We can without loss of generality start the analysis of the wave statistics evolution at the point when it enters Regime II.

In order to generalise the derivation of eq. (20) to broad-band seas, we use the definition of asymmetry from eq. (3). Consequently, the steepness in eqs. (15)-(20) will be corrected by the vertical asymmetry $\mathfrak{S}_{0}$, which in turn modifies the correction parameter:

$$
\Gamma_{\mathfrak{S}_{0}}\left(\varepsilon, k_{\mathrm{p}} h\right)=\frac{32+2 \tilde{\chi}_{1} \mathfrak{S}_{0}^{2} \pi^{2} \varepsilon^{2}}{32+\left(\tilde{\chi}_{1}+\chi_{1}\right) \mathfrak{S}_{0}^{2} \pi^{2} \varepsilon^{2}}
$$

The vertical asymmetry will increase the correction $\Gamma_{\mathfrak{S}_{0}}$ as compared to the narrow-band case by a few percent, as follows (see figure $1 \mathrm{~b}$ ):

$$
\frac{\Gamma_{\mathfrak{S}_{0}}\left(\varepsilon, k_{\mathrm{p}} h\right)}{\Gamma\left(\varepsilon, k_{\mathrm{p}} h\right)} \approx 1+\frac{1}{2}\left(\tilde{\chi}_{1}-\chi_{1}\right)\left(\mathfrak{S}_{0}^{2}-1\right)\left(\frac{\pi \varepsilon}{4}\right)^{2}+\mathcal{O}\left(\varepsilon^{4}\right)
$$

On the other hand, the parameter $\Gamma$ must be corrected for wave breaking, leading to slightly smaller peaks (see figure 2a). We include a depth-dependent breaking limit of regular waves [68] by setting $\varepsilon \leqslant\left(\varepsilon_{0} / 7\right) \tanh k_{\mathrm{p}} h$ with $0 \leqslant \varepsilon_{0} \leqslant 1$ :

$$
\Gamma_{\mathfrak{S}_{0}, 0} \approx \frac{1600+2 \pi^{2} \mathfrak{S}_{0}^{2} \varepsilon_{0}^{2} \tilde{\chi}_{1} \tanh ^{2} k_{\mathrm{p}} h}{1600+\pi^{2} \mathfrak{S}_{0}^{2} \varepsilon_{0}^{2}\left(\tilde{\chi}_{1}+\chi_{1}\right) \tanh ^{2} k_{\mathrm{p}} h}
$$



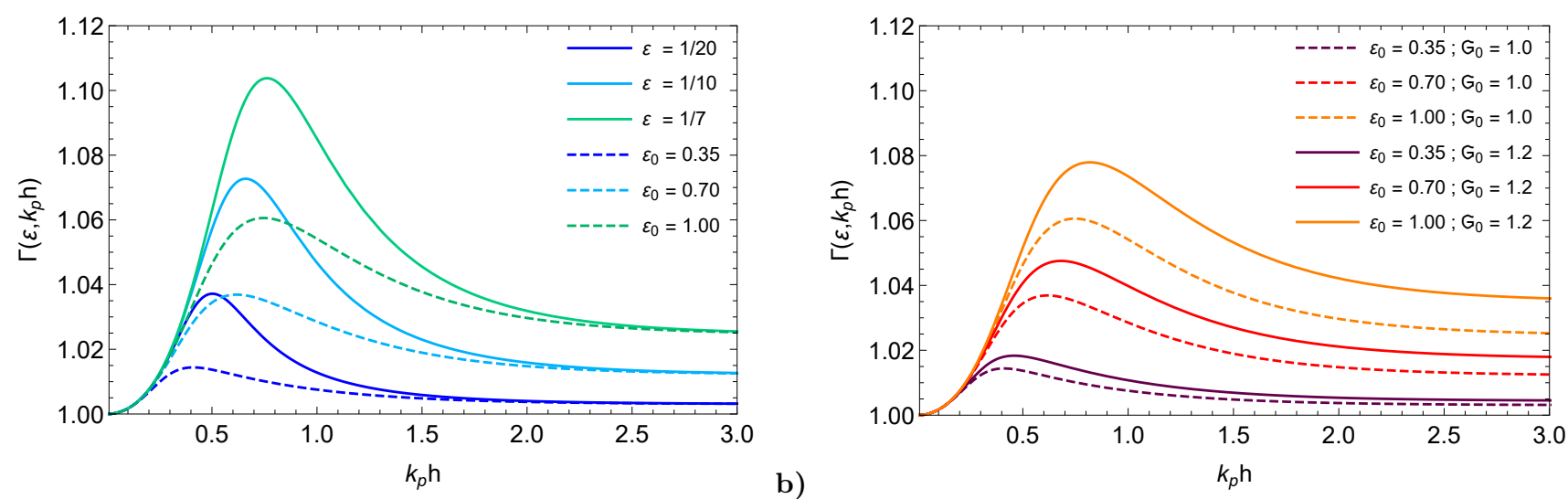

a)

b)

FIG. 2: $\Gamma$ correction parameter with the same initial significant steepness in deep water (a) with (dashed) or without wave breaking (solid) in narrow-banded seas, and (b) accounting for wave breaking in narrow-banded (dashed) and broad-banded seas (solid).

where even if the ratio $\varepsilon_{0}$ is constant, the actual (breaking-limited) steepness $\varepsilon$ will drop considerably throughout the transition from deep to shallow waters (see figure 2a). The vertical asymmetry $\mathfrak{S}_{0}$ increases the value of $\Gamma$ significantly (figure $2 \mathrm{~b}$ ), but less than the decrease in $\Gamma$ due to wave breaking. Although eq. (22) typically increases the shoal correction by a few percent, it also shows that the correction has an upper bound $\Gamma \leqslant 1.20$ when $\varepsilon_{0}=1$ and $k_{\mathrm{p}} h=0.5$ and maximum possible asymmetry $\mathfrak{S}_{0}=2$ (skewed sea with $\eta_{1 / 3} \approx 2.1$ ). Therefore, a decrease in the depth $k_{\mathrm{p}} h$ as in the experiments of Trulsen et al. [31] will increase the significant steepness as well as $\Gamma$, hence $\mathcal{R}_{\alpha, \Gamma}$ given in eq. (10), i.e. the probability of rogue waves as compared to the pre-shoal, homogenous Rayleigh distribution toward Regime II.

\section{B. The Normalised Height Diagram}

Since a bandwidth correction to the standard measure $H_{1 / 3}=4 \sigma$ will affect the Rayleigh distribution [54], such relation provides a valuable test for the Rayleigh distribution [69]. Hence, the distribution of wave heights contains information on the ratio $H_{1 / 3} / \sigma$, as follows:

$$
e^{-H^{2} / 8 m_{0}}=e^{-\frac{H^{2}}{8 H_{1 / 3}^{2}}\left(\frac{H_{1 / 3}}{\sqrt{m_{0}}}\right)^{2}} \equiv e^{-\frac{\alpha^{2}}{8}\left(\frac{H_{1 / 3}}{\sqrt{m_{0}}}\right)^{2}},
$$

which shows that at fixed energy (fixed $m_{0}=\sigma^{2}$ ) the change in the ratio $H_{1 / 3} / \sigma$ is balanced by the normalisation $\alpha=H / H_{1 / 3}$, therefore the overall exponent stays invariant, and thus the wave statistics. However, if and only if $m_{0}$ increases in comparison to Airy's solution, we expect the coefficient $H_{1 / 3}^{2} / m_{0}$ attached to $\alpha^{2}$ to be smaller. In fact, this can be seen by the ratio of the energies at first-order (6) and second-order (15). To investigate the effect of broad-band on $H_{1 / 3} / \sigma$ by the direct measurement of the vertical asymmetry as in eq. (3), we combine eqs. (10) and (24) and find (see figure 3a):

$$
H_{1 / 3}=\frac{4}{\mathfrak{S}_{0}} \sqrt{\frac{m_{0}}{\Gamma_{\mathfrak{S}_{0}}}}
$$

Consequently, the ratios $\frac{H_{1 / 3}}{\sqrt{m_{0}}}$ obtained from our model agree with the asymptotic values of 4 (narrow-banded in deep water, solid lines) and 3.8 (broad-banded in deep water, dashed line), as reported by Goda [69]. Asymmetry contributes to this expression via two different processes. On one hand, the $1 / \mathfrak{S}_{0}$ factor corresponds to its direct influence on the significant wave height. This factor does not depend on the occurrence of a shoal, although the asymmetry ultimately depends on the depth. Increasing the bandwidth and therefore $\mathfrak{S}_{0}$ will also lower $H_{1 / 3} / \sigma$ (see eq. (25)), as demonstrated by Vandever et al. [70]. On the other hand, the asymmetry influences the probability amplification by the shoal, via its impact on $\Gamma_{\mathfrak{S}_{0}}$. Except in very shallow water (Regime I), both $\mathfrak{S}_{0}$ and $\Gamma_{\mathfrak{S}_{0}}$ decrease monotonically as a function of $k_{p} h$, as we model the pre-shoal zone as a homogenous Airy solution concurrently leading to $\mathfrak{S}_{0} \rightarrow 1$ and $\Gamma_{\mathfrak{S}_{0}} \rightarrow 1$. We can therefore define a parameterisation mapping the latter on the former, in the form 

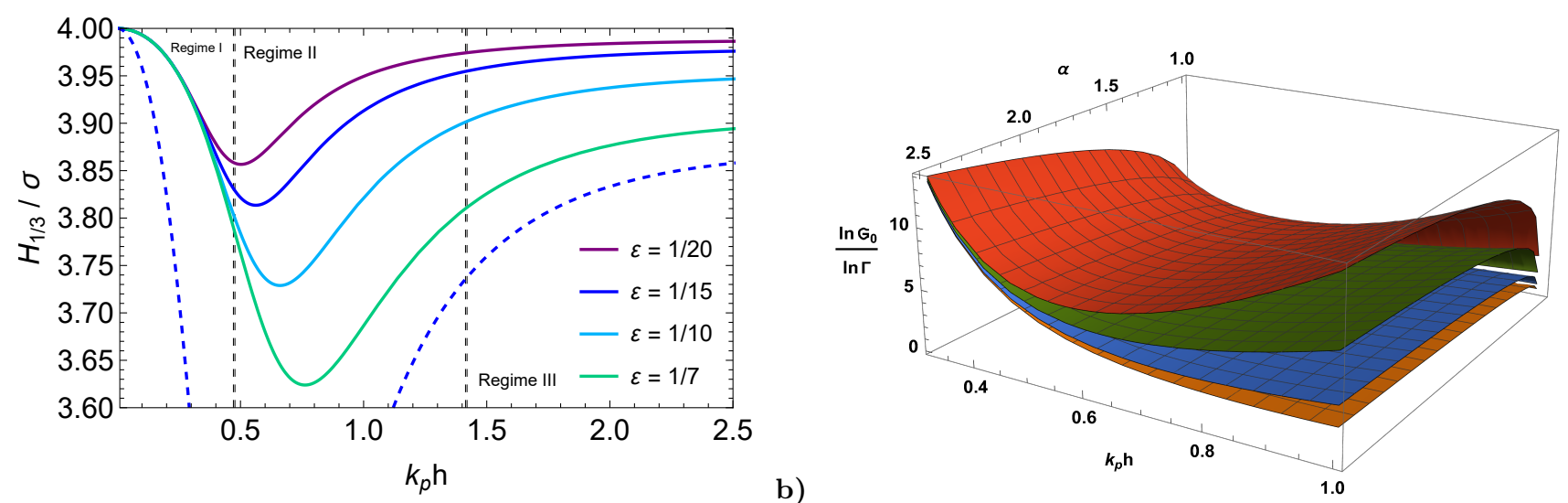

a)

b)

FIG. 3: (a) $H-\sigma$ diagram for narrow-banded $\mathfrak{S}_{0}=\sqrt{\Gamma}$ (solid) and otherwise with $\mathfrak{S}_{0} \sim \Gamma^{2}$ (dashed) for the specific case of $\varepsilon=1 / 15$ for comparison. (b) Ratio $\kappa=\ln \mathfrak{S}_{0} / \ln \Gamma$ with steepness: $1 / 7$ (orange), 1/10 (blue), 1/15 (green) and $1 / 20$ (red).

$\mathfrak{S}_{0}=\Gamma_{\mathfrak{S}_{0}}^{\kappa}$ (figure $\left.3 b\right)$, or, equivalently, its linear version $\mathfrak{S}_{0}=1+\kappa\left(\Gamma_{\mathfrak{S}_{0}}-1\right)$. Note that this parameterisation aims at describing the simultaneous evolution of $\Gamma$ and the asymmetry when the depth evolves, while eq. (22) only describes the direct impact of the asymmetry on $\Gamma$. Since Regime II is the one where we seek the evolution of the exceedance probability, the $\kappa$ parameterisation appears suitable to our work. Furthermore, its use avoids the numerical issues that would arise if handling $\mathfrak{S}_{0}$ and $\Gamma$ as independent.

\section{Evolution of a Narrow-Banded Arbitrary Probability Distribution}

So far we have studied a pre-shoal Rayleigh distribution of the surface elevation. Following eq. (10), the $\Gamma$ correction due to a rapid bathymetry change reads in this case:

$$
\begin{aligned}
\mathcal{R}_{\alpha, \Gamma}=e^{-2 \alpha^{2} / \Gamma}=\left(\mathcal{R}_{\alpha}\right)^{1 / \Gamma} \quad \therefore \quad \frac{\ln \mathcal{R}_{\alpha}}{\Gamma \ln \mathcal{R}_{\alpha, \Gamma}}=1, \\
\frac{\mathcal{R}_{\alpha, \Gamma}}{\mathcal{R}_{\alpha}}=\left(\mathcal{R}_{\alpha}\right)^{\left(\frac{1}{\Gamma}-1\right)}=e^{2 \alpha^{2}\left(1-\frac{1}{\Gamma}\right)} .
\end{aligned}
$$

The same correction therefore applies to any initial surface elevation distribution $\mathbb{P}$, as detailed in appendix C. For a narrow-banded sea:

$$
\mathbb{P}_{\alpha, \Gamma} \approx\left(\mathbb{P}_{\alpha}\right)^{\frac{1}{\Gamma}} \quad \therefore \quad \frac{\mathbb{P}_{\alpha, \Gamma}}{\mathbb{P}_{\alpha}} \approx e^{2 \alpha^{2}\left(1-\frac{1}{\Gamma}\right)}
$$

where $\mathbb{P}_{\alpha}$ denotes the exceedance probability at equilibrium prior to the shoal and $\mathbb{P}_{\alpha, \Gamma}$ is the distribution within the non-equilibrium zone. Hence, the highest possible realistic values of $\Gamma \sim 1.08$ (see figure 1 ) lead to a two-fold increase of rogue wave probability.

\section{Evolution of a Broad-Banded Arbitrary Probability Distribution}

In the case of a broad-banded sea, the connection between crest and height statistics responsible for eq. (25) can be reinterpreted as a simple change of variables $\Gamma \rightarrow \mathfrak{S}_{0}^{2} \Gamma_{\mathfrak{S}_{0}}$ in eq. (10), so that eq. (28) becomes:

$$
\mathbb{P}_{\alpha, \Gamma_{\mathfrak{S}_{0}}} \approx\left(\mathbb{P}_{\alpha}\right)^{\frac{1}{\mathfrak{S}_{0}^{2} \Gamma \mathfrak{S}_{0}}} \quad \therefore \quad \frac{\mathbb{P}_{\alpha, \Gamma_{\mathfrak{S}_{0}}}}{\mathbb{P}_{\alpha}} \approx e^{2 \alpha^{2}\left(1-\frac{1}{\mathfrak{S}_{0}^{2} \Gamma_{\mathfrak{S}_{0}}}\right)}
$$

To ensure the numerical stability of the distribution along the propagation, we use the $\kappa$ parameterisation introduced in section III B. For practical purposes, we estimate it as:

$$
\kappa_{0}=\frac{\ln \left[\max \mathfrak{S}_{0}\right]}{\ln \left[\max \Gamma\left(\langle\varepsilon\rangle, k_{p} h, \mathfrak{S}_{0}\right)\right]} \approx \frac{\ln \mathfrak{S}_{0}}{\ln \Gamma\left(\langle\varepsilon\rangle, k_{p} h, \mathfrak{S}_{0}\right)}
$$



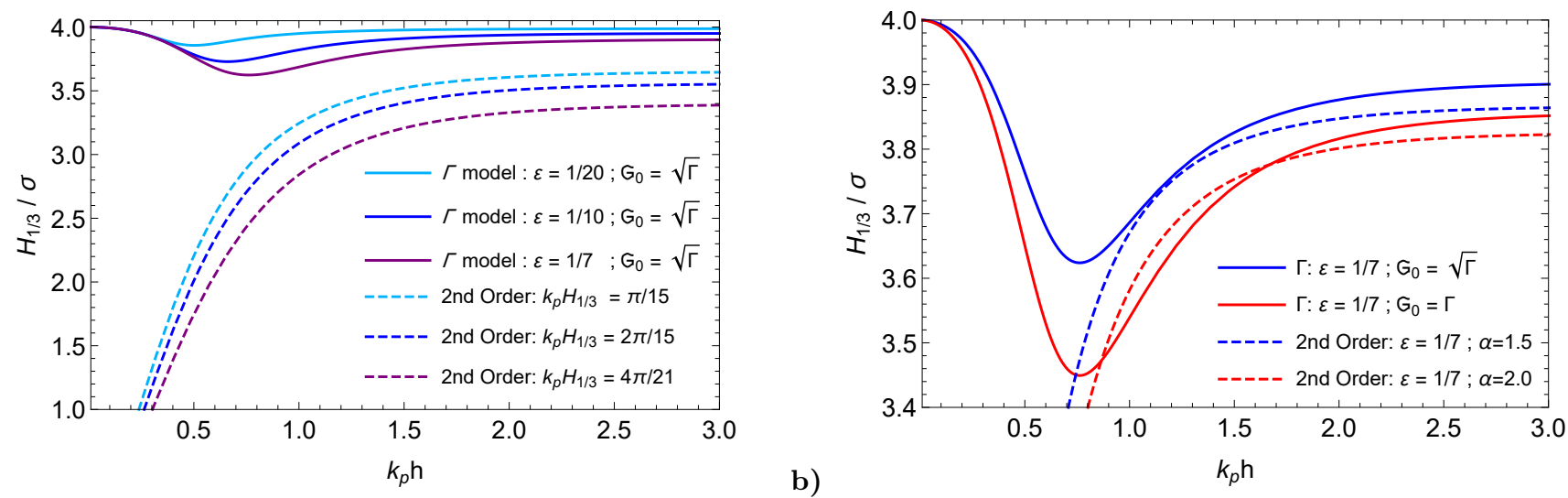

a)

b)

FIG. 4: (a) $\Gamma$-model (solid) as compared to the finite depth adjusted second-order structure (dashed) and (b) with corrected steepness.

where the maximum of $\Gamma$ is taken over the propagation on the whole shoal, $\langle\varepsilon\rangle$ being the average of the pre- and postshoal steepness. It can be measured at both stages of the wave propagation, or, alternately, obtained from forecast or hindcast, from the zero-crossing period and significant wave height. Note that uncertainties in asymmetry marginally impact the value of $\kappa_{0}$, as respective errors in the numerator and in $\Gamma$ tend to partially compensate each other due to eq. (22). Consequently, the effect of the shoal on the exceedance probability evolves during the propagation along $x$ as follows:

$$
\frac{\ln \mathbb{P}_{\alpha}}{\ln \mathbb{P}_{\alpha, \Gamma}}=\mathfrak{S}_{0}^{2} \cdot \Gamma=\left[\Gamma\left(\langle\varepsilon\rangle, k_{p} h, \mathfrak{S}_{0}\right)\right]^{2 \kappa_{0}} \cdot \Gamma\left(\varepsilon(x), k_{p} h(x), \mathfrak{S}_{0}\right)
$$

When the evolution spreads into Regimes I and III, the use of $\kappa_{0}$ is necessary to avoid divergence, though a continuous $\kappa$ restricted to Regime II is applicable. Note that under adequate conditions, a more compact form can be derived, as detailed in appendix D.

\section{E. Comparison with Standard Second-Order Models}

In this section we delineate similarities and differences between our model of second-order wave height probability and the typical treatment arising from Tayfun [42]. Following the second-order water surface, one finds [71]:

$$
\zeta(\phi=0):=\mathcal{Z}_{c}=\left[a \cos \phi+\frac{k a^{2}}{2} \cos (2 \phi)\right]_{\phi=0}=a+\frac{k a^{2}}{2}
$$

which normalised by the variance reduces to (with nomenclature $\sigma \tilde{X}:=X$ ):

$$
\tilde{\mathcal{Z}}_{c}=\tilde{a}+\tilde{a}^{2} \cdot \frac{k \sigma}{2} \quad \therefore \quad \tilde{a}=\frac{\sqrt{1+2 \tilde{\mathcal{Z}}_{c} \sigma k}-1}{\sigma k} .
$$

Applying the finite depth coefficients in eq. (2.382a) of Dingemans [63], one finds:

$$
\tilde{\mathcal{Z}}_{c}=\tilde{a}+\tilde{a}^{2} \cdot \frac{k \sigma}{2} \cdot \mathcal{F} \quad, \quad \mathcal{F}=\mathcal{F}(k h)=\frac{3-\tanh ^{2} k h}{2 \tanh ^{3} k h},
$$

leading to the mathematical structure (see Mendes et al. [18] for the $\mathcal{F}=1$ case):

$$
\mathbb{P}\left(H>\alpha H_{1 / 3}\right)=\exp \left\{-\frac{8}{k^{2} H_{1 / 3}^{2} \mathcal{F}^{2}}\left[\sqrt{1+\alpha k H_{1 / 3} \mathcal{F}}-1\right]^{2}\right\} .
$$

Note, however, that this distribution was not derived in the narrow-band model of Tayfun [42], it is rather an adaptation to extract the mathematical structure of a probability growing boundlessly with increasing steepness. 
Although Tayfun [48] puts limitations to the steepness as $\mu_{3} \lesssim k H_{1 / 3} /\left(1+\nu^{2}\right)$, North Sea data [15, 18] shows that the skewness can grow higher. Therefore, the suggested adaptation to wave heights is usefull since now $\varepsilon$ can reach its breaking limit [68]. In figure 4a we compare our $\Gamma$ model of eq. (29) with that of eq. (35) through the $H-\sigma$ diagram:

$$
\frac{H_{1 / 3}}{\sqrt{m_{0}}}=\frac{8}{\alpha k H_{1 / 3} \mathcal{F}}\left[\sqrt{1+\alpha k H_{1 / 3} \mathcal{F}}-1\right]
$$

The finite-depth-adapted height model yields 10-20\% lower ratios in deep water and drops towards zero in shallow water, in contradiction with observations [69]. Nonetheless, our model can be consistent with the structure of eq. (35) in both deep and transitional waters $\left(k_{p} h \geqslant 0.8\right)$ for moderate values of $\alpha$ and within the narrow-band validity of Tayfun [42], provided eq. (35) is corrected to the steepness $k_{\mathrm{p}} H_{1 / 3} \rightarrow \varepsilon_{s}$, where $\varepsilon_{s}:=\left\langle\varepsilon_{i}\right\rangle_{r}=\left\langle H_{1 / 3} / \lambda_{i}\right\rangle_{r}$, see figure 4b. Indeed, we can use $\left\langle\lambda_{i}\right\rangle_{r} \approx 1.5 \bar{\lambda}$ [18], which implies $\varepsilon=H_{1 / 3} / \bar{\lambda} \approx 1.5 \varepsilon_{s} \approx 3 k H_{1 / 3} / 4 \pi$. For transitional and especially shallower regions reaching the limit of the second-order approximation, the above adapted model would not reproduce the same probability amplification as our model, nor the original one [42]. Hence, our model departs from the Tayfun [42] in finite depth and recovers it in deep water.

\section{COMPARISON WITH TRULSEN EXPERIMENTS}

Raustøl [26] provides experiments of wave propagation over a shoal, later summarised in Trulsen et al. [31]. These experiments were carried out in a $24.6 \mathrm{~m}$ long and $0.5 \mathrm{~m}$ wide wave tank. Surface elevation measurements were made with ultrasound probes and velocity measurements were made with an Acoustic Doppler Velocimeter, with an array of 16 probes that was moved to four different locations such that the resolution before the shoal was $0.3 \mathrm{~m}$ and the resolution above the shoal was $0.1 \mathrm{~m}$. The wavemaker generated a JONSWAP spectrum with peak parameter $\gamma=3.3$, with typical peak periods $T_{p} \sim 1 \mathrm{~s}$. The probability distribution of rogue waves evolving with the distance from the wavemaker was recorded, offering a benchmark for our non-homogenous correction to the wave height probability distribution. Since our model is expressed in terms of dimensionless depth and significant steepness, we have extracted the raw data from Figures 5.4-5.5 of Raustøl [26], in accordance with the inversion $k_{\mathrm{p}} h \approx\left(k_{\mathrm{p}} a_{c} / \mathrm{Ur}\right)^{1 / 3}$ of Trulsen et al. [31]. In order to facilitate the processing, we have smoothed the experimental data by fitting analytic functions on the data points, as described in detail in appendix E. The experiments feature a shoal starting at $x=0$, rising up to $42 \mathrm{~cm}$ at $x=1.6 \mathrm{~m}$, followed by a plateau till $x=3.2 \mathrm{~m}$ and a decay to zero until $x=4.8 \mathrm{~m}$. The initial depth ranges between 50 and $60 \mathrm{~cm}$ depending on the runs, as detailed in table 1 of Trulsen et al. [31] and table 5.2 of Raustøl [26].

\section{A. Results}

Based on the $\kappa_{0}$ parameterisation described in the previous section, we compute the evolution of the rogue-wave probability as a function of distance for each run, assuming a pre-shoal homogenous Rayleigh distribution (figure 5). The values of $\kappa_{0}$ calculated according to eq. (30) amount to $(5.9,4.9,4.3,3.6,2.9,2.6,2.3,2.4,2.3,2.2)$ for the runs 1-2, 4-9 and 11-12, respectively. Our model that takes into account the evolution of the wave asymmetry $\mathfrak{S}_{0}$ over the shoal (cyan curves) due to the skewness of the surface elevation distribution (eq. (3)) reproduces well the experimental data, over the whole shoaling episode and for all runs. Disregarding the evolution of skewness reported in Trulsen et al. [31] while keeping a vertical fixed asymmetry $\mathfrak{S}_{0}=1.2$ (blue curves in figure 5) degrades the agreement only marginally, although the probability rises slightly earlier, decays slightly later, and the asymmetry between the upand down-shoaling phases is reduced. Furthermore, we point out the remarkable difference of amplification between vertically asymmetrical (solid blue, cyan) and symmetrical seas (dashed red). This happens because eq. (26) leads to a maximal amplification between $75-100 \%$ for $\Gamma \approx 1.08-1.10$. When we include the typical vertical asymmetry $\mathfrak{S}_{0}^{2} \sim 1.5$, the pre-shoal probability $\mathbb{P}_{\alpha}$ is transformed into $\mathbb{P}_{\alpha}^{2 / 3} \sim 10 \mathbb{P}_{\alpha}$ within Regime II, seemingly becoming an alternative to Gram-Charlier models [72]. Finally, adjusting the homogenous pre-shoal probability to the observed values instead of considering an initial Rayleigh distribution (dotted curve) improves the agreement (runs 6-12, panels e-j), demonstrating the applicability of our model to arbitrary probability distributions. This agreement over the whole range of experimental conditions reported by Raustøl [26] is remarkable, as it requires no specific parameter tweaking. 


\section{B. Discussion}

In contrast, Tayfun [42] (see the discussion following eqs. (36)-(38) of that paper) claims a Gaussian probability distribution for the second-order wave heights, as also discussed in Tayfun [73], Tayfun and Fedele [74]. In fact, these models fall under the broad category of quasi-determinism theories [9] of which Longuet-Higgins [54] and Naess [75] also take part and preclude wave heights from exceeding the Rayleigh distribution, typically being lower than the latter as the bandwidth broadens. Therefore, these formulations in homogenous conditions would not be able to describe the Raust $\varnothing 1$ [26], Trulsen et al. [31] experiments. Also according to these models, any departure from a Rayleigh distribution of wave heights is due to third-order nonlinearities [74, 76]. Nevertheless, it is important to remark that most of these theories were devised for deep waters, hence they fall within the Regime III of our model. Moreover, following Dingemans [63], Marthinsen [77] one can tentatively elaborate alternative finite-depth secondorder mathematical structures as in section III E. Comparing with our model, we observe a numerical equivalence in deep water, but not in transitional and shallow waters where the alternative second-order models display a sharp departure from Goda [69] observations. Unfortunately, the finite depth model of Tayfun and Alkhalidi [78] is not a distribution of wave heights, hence not applicable to our discussion.

Our $\Gamma$-model of the probability evolution over a shoal is based on the second-order correction, which is valid for $\mathrm{Ur} \leqslant 8 \pi^{2} / 3$ [49]. This limit of validity is well beyond the Ursell number $\mathrm{Ur} \leqslant 0.22$ of the experiments considered in this work $[26,31]$. We can therefore expect that the $\Gamma$-model will still apply to more than 10 times larger waves and/or shallower waters.

The transient drop of $\mathbb{P}_{\alpha, \Gamma} / \mathbb{P}_{\alpha}$ to almost zero in the rising region of the shoal $(0 \leqslant x \leq 1.6 \mathrm{~m})$ in runs $1,2,4$, and 5 , as well as its slow decay on the trailing side $(3.2 \leq x \leq 4.8 \mathrm{~m})$ could be due to higher-order nonlinear effects not captured in our model, including the evolution of the skewness and kurtosis of the surface elevation probability distribution when propagating on the shoal.

Recent developments in Zhang and Benoit [36] described the exceedance probability of Run 3 of Trulsen et al. [31] throughout the shoaling and de-shoaling stages by means of numerical simulations. However, an analytical expression for the probability distribution was not provided, and cases with low or vanishing skewness and kurtosis like Run 12 were not addressed. Our model, provides an explicit expression with no free fitting parameter. It is directly obtained from second-order correction and has shown to be valid for all runs in Raustøl [26]. Although we were not able to analyse Run 3 [79] due to the lack of wave height probability data, we are confident that our model can reproduce it well, as it does for the very similar Runs 1,2, and 4.

The modulational instability can not account for the rise of the rogue wave probability due to shoaling in the considered experiments because they do not feature narrow-banded Stokes waves nor lie in the optimal range $k_{p} h>$ 1.36 [8] in the shallower side, except for runs 11 and 12 that showed no significant amplification. On the other hand, all runs start in the range $k_{p} h>1.36$ before the shoal and showed no large deviation from the Gaussian sea except for Run 12 .

Furthermore, our model and in particular the three regimes discussed on eq. (20) and figure $1 \mathrm{~b}$ also allow to understand the contradictory behaviours highlighted by Trulsen et al. [31] and Zhang and Benoit [36]. While the rogue wave probability is not affected by a shoal in initially deep water (Regime III), it does increase on a shoal in intermediate depth (Regime II). Interestingly, a third regime (Regime I of figure 1b) is consistent with observed surf zone statistics [16], i.e. lower rogue wave likelihood than in transitional waters. In addition, Barbariol et al. [80] shows an increase of maximum crest up to some cut-off in dimensionless depth, upon which it starts to sharply decrease the higher the ratio $H_{1 / 3} / h$ becomes, thus providing support for Regime I in our model. However, numerical simulations and experiments with even shallower shoals are necessary for a conclusive assessment. Therefore, we have demonstrated that rogue wave statistics will be enhanced by non-equilibrium dynamics of rapid depth change for a dimensionless depth $0.5<k_{p} h<1.5$ but stops growing when it leaves this range. It is therefore less pronounced past the threshold $k_{\mathrm{p}} h \leqslant 0.3$ until it starts to follow shallow water distributions such as Glukhovskii [16].

The evolution of the rogue wave probability highlighted by our model could be related to the process proposed by $\mathrm{Li}$ et al. [40] and experimentally confirmed in $\mathrm{Li}$ et al. [39], in which the generation of additional wave packets that interact with the original pre-shoal wave packet propagating over a step leads to a local peak some distance into the shallower region. Notably, Li et al. [40] have used a more general treatment for the surface elevation, containing both super and sub-harmonics, as well as free and bound waves. Moreover, instead of bound super-harmonic in our model, one could investigate the effect of the nonlinear evolution of interacting free modes. For instance, similar experimental results were interpreted using a statistical matching of KdV equilibrium states at the depth transition point [37, 38]. In retrospect to the ideas in Onorato and Suret [81], these works obtained a connection between variance and skeweness with the dynamics. Therefore, subsequent investigations carrying out a comparison between the two types (free and bound) of mode evolution is of great relevance. 


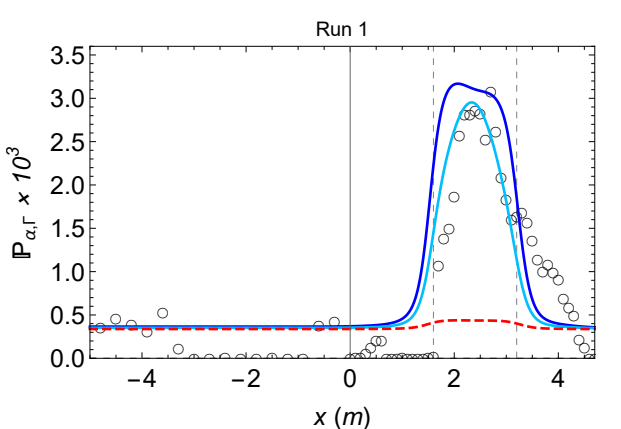

a)

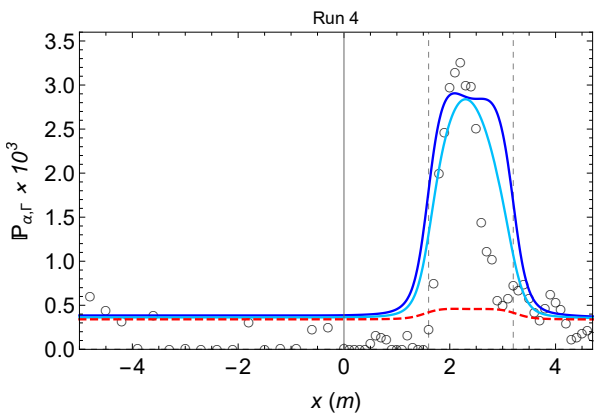

c)

e)

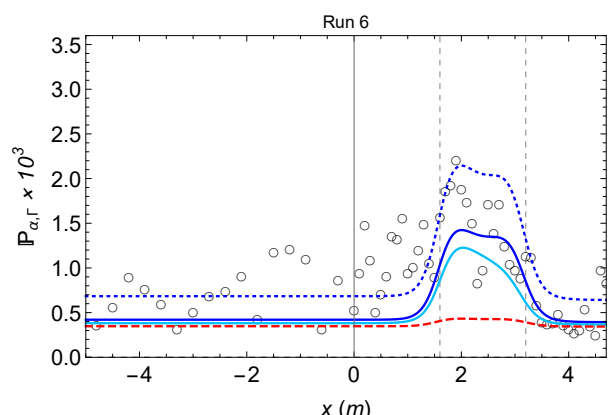

g)
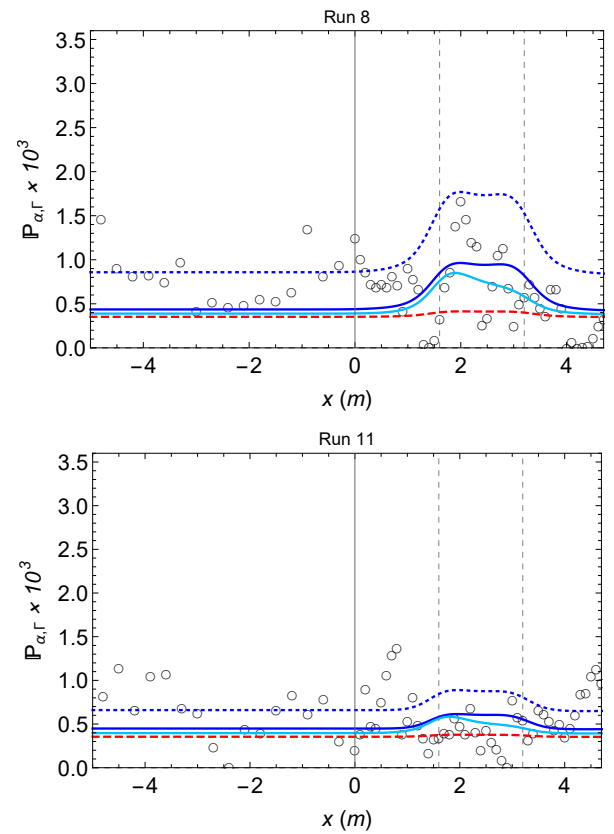

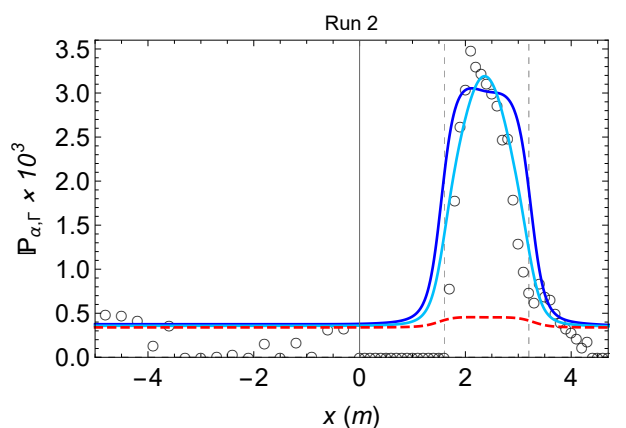

b)

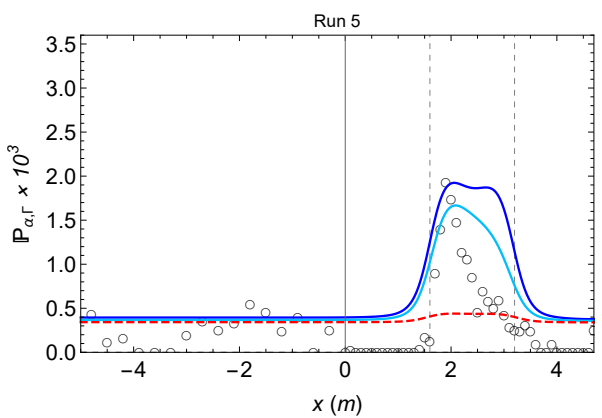

d)

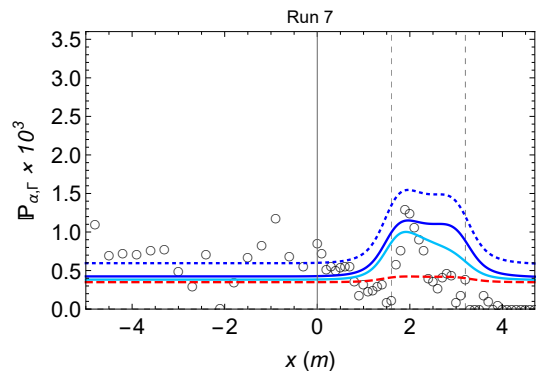

f)

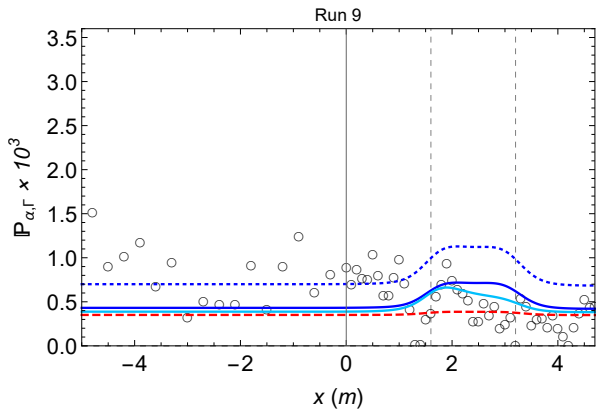

h)

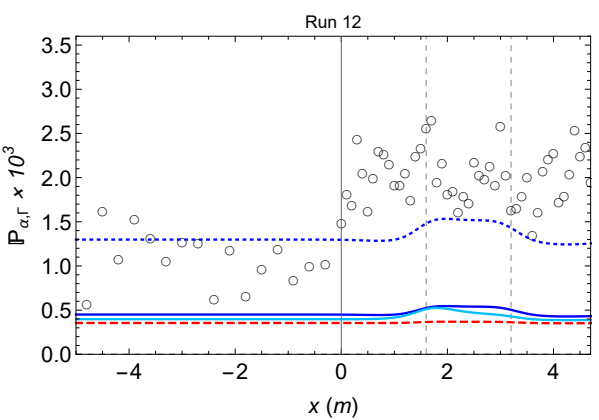

FIG. 5: Evolution of the probability of rogue waves $(\alpha \geq 2)$ over a shoal for Runs 1-2, 4-9, 11-12 from figure 5.8 of Raust $\varnothing$ [ [26]. Blue: Model of eq. $(28)$ for a pre-shoal $(x<0)$ Rayleigh distribution with $\mathfrak{S}_{0}=1.2$; Cyan: Same model considering the evolution of skewness over the shoal; Dotted: Model of eq.(28) for a pre-shoal probability matched to the experimental data. Dashed: same as blue but for symmetrical seas $\mathfrak{S}_{0}=1$. 


\section{CONCLUSION}

This work presented a connection between statistical distributions and the fluid mechanics of the second-order perturbation in non-homogenous conditions, successfully providing a physical explanation for the rogue wave probability increase over a depth change [26,31]. We have shown that our model reproduces very well the experiments of Raustøl [26], Trulsen et al. [31] regarding the probability distribution as a function of the distance from the wavemaker. Moreover, we showed that the significant steepness and dimensionless depth affect the validity and numerically assessed the extent of the deviation from the assumption of homogeneity. Furthermore, instead of introducing new physics [23], our model has demonstrated that an effective theory arises by challenging the homogeneity assumption. Introducing the $H_{1 / 3} / \sigma$ ratio [69], we have established that the deep water regime produces no significant amplification of the height distribution, whereas the transitional water within $0.5<k_{p} h<1.5$ provides strong amplification, and the shallow water regime decreases this large amplification to a level smaller than the initial stage in deep water.

Our model has been restricted to reformulating moments up to the variance only. The evolution of either skewness or kurtosis as a function of the distance from the wavemaker [31] will be addressed in a subsequent work. On the other hand, we have shown that it is possible to fit the data without the application of either skewness nor kurtosis, as they are 'symptoms' of the dynamics and not the cause [15, 82, 83]. Since our model relies on relations between steepness, slope and bandwidth that might be affected by a rapid depth change, the empirical findings in Mendes et al. [18] regarding vertical asymmetry have to be extended to the current setting for an exact formulation. Furthermore, the generalisation of this work to multidirectional spectra would be needed, since Ducrozet and Gouin [34] suggest that such configuration weakens the effect of a varying bathymetry. Whether rogue waves are enhanced in strong bathymetry changes throughout most oceans or regionally under suitable conditions is yet to be assessed.

\section{ACKNOWLEDGEMENTS}

S.M, M.B. and J.K. were supported by the Swiss National Science Foundation under grant 200020-175697. S.M. and A.S. were supported by the National Science Foundation under grant OCE-1558978.

Declaration of Interests. The authors report no conflict of interest.

\section{Appendix A: Spectral Analysis of Spatial and Temporal Series}

Below we discuss two aspects of the assumptions necessary to obtain a sea with Gaussian statistics, the first dealing with a steady-state treatment of the statistical moments and the latter dealing with otherwise out-of-equilibrium conditions.

\section{General Considerations}

Firstly, however, it is important to highlight the difference between stationarity and homogeneity. Following the classical definitions, they typically mean non-equilibrium series in regard to translations in time and space, respectively. Traditionally, the Khintchine [51] theorem (KT) is often written for the time domain only, but it can be easily generalized to the spatial domain due to their symmetry $[84,85]$. Indeed, the spatial KT has the same structure, only replacing the time lag by a displacement vector [86-88].

Under general conditions of quasi mean-ergodic processes (i.e. the ensemble average of the surface elevation is not zero but very small as compared to the second moment, see appendices A 2 and C), the autocorrelation of the sea surface elevation, which by definition is computed from the ensemble average, can be properly computed from the spatial average $\langle\cdot\rangle$ :

$$
R_{x}(\xi):=\mathbb{E}[\zeta(x) \zeta(x+\xi)] \approx\langle\zeta(x) \zeta(x+\xi)\rangle \therefore R_{x}(0) \equiv\left\langle\zeta^{2}\right\rangle=\lim _{L \rightarrow+\infty} \frac{1}{L} \int_{0}^{L} \zeta^{2}(x) d x
$$

such that one can find $[9,53]$ :

$$
R_{x}(\xi)=\frac{1}{2} \sum_{i} a_{i}^{2} \cos \left(k_{i} \xi\right) \quad \therefore \quad R_{x}(0)=\left\langle\zeta^{2}\right\rangle=\frac{1}{2} \sum_{i} a_{i}^{2}=\mathscr{E}_{A I R Y}
$$


The above result guarantees that the autocorrelation forms a Fourier pair with the spatial energy spectrum. However, by definition (see eq. (A4)), the distribution for the surface elevation $\zeta$ has to be extracted from its ensemble moments. Unless one assumes a uniform distribution of phases (see appendix A 2), the above rationale is unable to provide ensemble moments, but do provide exact values for the spatial moments. Therefore, as demonstrated by appendix A 2, a sufficiently large spatial series with uniform distribution of phases leads to:

$$
\mathbb{E}[\zeta]=\langle\zeta\rangle=0 \quad, \quad \mathbb{E}\left[\zeta^{2}\right]=\left\langle\zeta^{2}\right\rangle=\frac{1}{2} \sum_{i} a_{i}^{2}
$$

thus narrowing down the possible solutions into the Gaussian distribution of the surface elevation because of the ensemble moments $\mathbb{E}\left[\zeta^{2 n+1}\right]=0$ for all $n \in \mathbb{N}$ and vanishing excess kurtosis. This procedure is very common in complex physical systems and the above property is called ergodicity, which for time domains have been known since Boltzmann [89]. Despite the simplification brought by ergodic theory in physical systems of high complexity, proving its validity is always a challenging task [90]. Hence, wave statistics models have to assume homogeneity and ergodicity in order to find results similar to eq. (A3).

\section{Random Phase Distribution}

The exact computation of the ensemble average of the sea surface elevation at an instant of time $t_{0}$ is not trivial [53]. Without ergodicity, the probability density of the surface elevation is unknown unless one assumes its expected value:

$$
\mathbb{E}[\zeta]=\int \zeta d \mu(\zeta)
$$

where $d \mu(\zeta)$ is a measure on the space of possible surface elevations. For an oscillatory system, it is customary to write $\zeta=r \cos (\phi)$ and introduce a joint $\mathrm{PDF}$ on the space $[0, \infty) \times[0,2 \pi)$ that describes the statistical distribution of amplitudes and phases. Without loss of generality, we can always choose units in which $\mathbb{E}[\zeta]=1$, and write:

$$
\mathrm{PDF}=\frac{f(r, \phi)}{2 \pi} r e^{-r^{2} / 2} d r d \phi
$$

Longuet-Higgins [12] distribution is recovered if we assume that the phases are uniformly distributed and uncorrelated from the amplitudes, that is, $f(r, \phi)=1[3,13,72,91]$. However, the uniform distribution of phases is only appropriate for narrow-banded signals [92, 93]. For more realistic sea states, Tayfun [56] shows that the PDF introduced above should be corrected to account for correlations between phase and amplitude. As a first approximation, the correction proposed by Tayfun [56] is:

$$
f(r, \phi)=1+\frac{\mu_{3}}{6} r\left(r^{2}-4\right) \cos \phi
$$

where $\mu_{3}$ is the skewness. Note that within this approximation, the expected value of even order powers of $\zeta$ are not modified relative to the uniform phase approximation. Odd order powers, which are zero when $f=1$, are now non zero. On the other hand, it is known that the weakly nonlinear evolution of a sea state which at $t=0$ has random and uniformly distributed independent phases, remains so over the nonlinear time interval $t \gtrsim 2 \pi / \omega_{p}$ $[94,95]$. Therefore, the source of correlations between phases and amplitudes cannot be attributed to the internal weakly nonlinear dynamics. However, this does not preclude that external factors (e.g., wind forcing) inducing nonequilibrium dynamics can nudge the phase distribution away from uniformity and/or impart a correlation between phases and amplitudes.

For the purpose of illustration, let us analyse the simplest effect of a uniform distribution. Through a change of variables [96] and the law of the unconscious statistician [97], we rewrite the ensemble average:

$$
\mathbb{E}[\zeta]=\int_{-\infty}^{+\infty} \zeta f(\zeta) d \zeta=\int_{0}^{2 \pi} \zeta(\phi) f(\phi) d \phi
$$

Therefore, the uniform distribution of phases leads to ergodicity:

$$
\mathbb{E}[\zeta]=\sum_{i} \frac{a_{i}}{2 \pi} \int_{0}^{2 \pi} \cos \phi d \phi=\langle\zeta(x)\rangle=\lim _{L \rightarrow+\infty} \sum_{i} \frac{a_{i}}{L} \int_{0}^{L} \cos \left(k_{i} x\right) d x=0
$$




$$
\mathbb{E}\left[\zeta^{2}\right]=\sum_{i} \frac{a_{i}^{2}}{2 \pi} \int_{0}^{2 \pi} \cos ^{2} \phi d \phi=\left\langle\zeta^{2}(x)\right\rangle=\lim _{L \rightarrow+\infty} \sum_{i} \frac{a_{i}^{2}}{L} \int_{0}^{L} \cos ^{2}\left(k_{i} x\right) d x=\sum_{i} \frac{a_{i}^{2}}{2}
$$

If we assume that correlations develop between the phases, a weak departure from ergodicity will be observed. Below we show that, due to the ergodicity assumption, the accuracy of Gaussian statistics will deteriorate the narrower the superposition distribution is. For the sake of measuring appreciable deviations and without loss of generality, we use a Boltzmann-like distribution, such that the ensemble average of the sea surface reads:

$$
\mathbb{E}^{(B)}[\zeta]=\sum_{i}\left[\frac{a_{i}}{\pi} \int_{0}^{+\infty} \frac{\cos \phi}{e^{\phi / \pi}} d \phi\right]=\sum_{i} \frac{a_{i}}{\left(1+\pi^{2}\right)} \sim 0.2 \sqrt{m_{0}}
$$

which is relatively small compared to the second moment of the surface elevation. For a tentative Gaussian shaped superposition, however, one finds:

$$
\mathbb{E}^{(G)}[\zeta] \approx \sum_{i} a_{i}\left[\frac{3}{5} \int_{0}^{+\infty} \cos \phi e^{-(\phi-1)^{2}} d \phi\right]=\sum_{i} \frac{3 a_{i}}{8} \sim 0.8 \sqrt{m_{0}}
$$

while for the square of the surface elevation we obtain:

$$
\begin{aligned}
\mathbb{E}^{(B)}\left[\zeta^{2}\right] & \approx \sum_{i} \frac{a_{i}^{2}}{\pi} \int_{0}^{+\infty} e^{-\phi / \pi} \cos ^{2} \phi d \phi+\sum_{i \neq j} \frac{a_{i} a_{j}}{\pi} \int_{0}^{+\infty} e^{-\phi / \pi} \cos \phi d \phi \\
& \approx\left(\frac{1+2 \pi^{2}}{1+4 \pi^{2}}\right) \sum_{i} a_{i}^{2}+\sum_{i \neq j} \frac{a_{i} a_{j}}{1+\pi^{2}} \sim\left(\frac{4+14 \pi^{2}+4 \pi^{4}}{1+5 \pi^{2}+4 \pi^{4}}\right) m_{0} \sim 1.2 m_{0}
\end{aligned}
$$

Lacking a closed-form, the Gaussian-like distribution of phases reads instead:

$$
\mathbb{E}^{(G)}\left[\zeta^{2}\right] \approx \sum_{i} \frac{5 a_{i}^{2}}{13}+\sum_{i \neq j} \frac{3 a_{i} a_{j}}{8} \sim 1.53 m_{0}
$$

We compare the deviations from the uniform superposition in eqs. (A10)-(A12):

$$
\delta \mathbb{E}_{1,2}^{(B)}=\frac{\sqrt{\mathbb{E}^{(B)}\left[\zeta^{2}\right]-\mathbb{E}^{(U)}\left[\zeta^{2}\right]}}{\mathbb{E}^{(B)}[\zeta]-\mathbb{E}^{(U)}[\zeta]}=\frac{\sqrt{1.2 m_{0}-m_{0}}}{0.2 \sqrt{m_{0}}-0} \approx 2.3
$$

whereas for the Gaussian one we have:

$$
\delta \mathbb{E}_{1,2}^{(G)}=\frac{\sqrt{\mathbb{E}^{(G)}\left[\zeta^{2}\right]-\mathbb{E}^{(U)}\left[\zeta^{2}\right]}}{\mathbb{E}^{(G)}[\zeta]-\mathbb{E}^{(U)}[\zeta]}=\frac{\sqrt{1.53 m_{0}-m_{0}}}{0.8 \sqrt{m_{0}}-0} \approx 0.9
$$

implying a decreasing gap between moments when the superposition distribution is narrower. In these examples, we see that even the linear evolution of a sea state that, at some point in time, has a non-uniform distribution of phases breaks ergodicity. As a remark, the break in ergodicity and Gaussianity are not necessarily simultaneous, as in the above case.

\section{Appendix B: Energetic Formulae Derivation}

Given eq. (11) and taking the limit of very large number of amplitude components towards an asymptotic leading order, the energy computation is reduced to the coefficients $\left(\Omega_{m}, \widetilde{\Omega}_{m}\right)$. Then, having $u_{i}=\partial \Phi / \partial x_{i}$ and using the 
notation $I=u_{1}^{2}+u_{3}^{2}$, one obtains:

$$
\begin{aligned}
I & =\left[\frac{\partial}{\partial x}\left\{\sum_{j} f_{j} \cosh (j \varphi) \sin (j \phi)\right\}\right]^{2}+\left[\frac{\partial}{\partial z}\left\{\sum_{j} f_{j} \cosh (j \varphi) \sin (j \phi)\right\}\right]^{2} \\
& =\left[\sum_{j} j k \cdot f_{j} \cosh (j \varphi) \cos (j \phi)\right]^{2}+\left[\sum_{j} j k \cdot f_{j} \sinh (j \varphi) \sin (j \phi)\right]^{2} \\
& =\left[\sum_{m} \Omega_{m} \cosh (m \varphi) \cos (m \phi)\right]\left[\sum_{n} \Omega_{n} \cosh (n \varphi) \cos (n \phi)\right] \\
& +\left[\sum_{m} \Omega_{m} \sinh (m \varphi) \sin (m \phi)\right]\left[\sum_{n} \Omega_{n} \sinh (n \varphi) \sin (n \phi)\right]
\end{aligned}
$$

where we defined $\Omega_{m}=m k f_{m}$ and estimated the effect of $|\partial h / \partial x|$ to not be of leading order for this sum (see sections III and IV B). By means of the notation:

$$
\operatorname{Cos}_{m n}(\varphi, \phi):=\cosh (m \varphi) \cdot \cos (n \phi) \quad ; \quad \operatorname{Sin}_{m n}(\varphi, \phi):=\sinh (m \varphi) \cdot \sin (n \phi),
$$

the algebra yields:

$$
\begin{aligned}
I & =\sum_{m=n} \Omega_{m}^{2} \mathfrak{C o s}_{m m}^{2}(\varphi, \phi)+\sum_{m \neq n} \Omega_{m} \Omega_{n} \mathfrak{C o s}_{m m}(\varphi, \phi) \mathfrak{C o s}_{n n}(\varphi, \phi) \\
& +\sum_{m=n} \Omega_{m}^{2} \mathfrak{S i n}_{m m}^{2}(\varphi, \phi)+\sum_{m \neq n} \Omega_{m} \Omega_{n} \mathfrak{S i n}_{m m}(\varphi, \phi) \mathfrak{S i n}_{n n}(\varphi, \phi) \\
& :=\sum_{m} \Omega_{m}^{2} I_{m m}+\sum_{m \neq n} \Omega_{m} \Omega_{n} I_{m n} .
\end{aligned}
$$

However, one can further expand the trigonometric clusters in eq. (B2) as follows:

$$
\begin{aligned}
4 I_{m n} & =4 \mathfrak{C o s}_{m m}(\varphi \phi) \mathfrak{C o s}_{n n}(\varphi \phi)+4 \mathfrak{S i n}_{m m}(\varphi \phi) \mathfrak{S i n}_{n n}(\varphi \phi), \\
& =[\cos (m \phi) \cos (n \phi)+\sin (m \phi) \sin (n \phi)] \cdot 2 \cosh [(m+n) \varphi] \\
& +[\cos (m \phi) \cos (n \phi)-\sin (m \phi) \sin (n \phi)] \cdot 2 \cosh [(m-n) \varphi], \\
& =2 \cosh [(m+n) \varphi] \cos [(m-n) \phi]+2 \cosh [(m-n) \varphi] \cos [(m+n) \phi] .
\end{aligned}
$$

As an immediate corollary, we find $2 I_{m m}=\cosh (2 m \varphi)+\cos (2 m \phi)$. Using the algebra from eq. (B4) and periodic integration, following the expression for the energy in eq. (4) and subtracting the potential energy $\rho g h_{0}^{2} / 2$ due to the water column, we find in the limit $i \rightarrow \infty$ the leading order energy density:

$$
\mathscr{E} \approx \sum_{m} \frac{\tilde{\Omega}_{m}^{2}}{4}+\sum_{m} \frac{\Omega_{m}^{2}}{4 g} \int_{-h}^{0} \cosh (2 m \varphi) d z \approx \frac{1}{4} \sum_{m}\left[\tilde{\Omega}_{m}^{2}+\Omega_{m}^{2} \cdot \frac{\sinh (2 m k h)}{2 m g k}\right] .
$$

As we assumed a small effect of $\partial h / \partial x$ in the previous integral, the bathymetry will appear in $\varphi=k(z+h)$ as well as in $\Omega_{m}$ and $\tilde{\Omega}_{m}$. Likewise, using the definition of eq. (11), we compute the spatial average of the squared sea surface elevation:

$$
\left\langle\zeta^{2}\right\rangle=\lim _{L \rightarrow+\infty} \frac{1}{L} \int_{0}^{L}\left[\sum_{m} \tilde{\Omega}_{m} \cos (m \phi)\right]\left[\sum_{n} \tilde{\Omega}_{n} \cos (n \phi)\right] d x=\sum_{m} \frac{\tilde{\Omega}_{m}^{2}}{2} .
$$

To compare the generalised model with the specific case of Airy's solution we set $m=1$. In this case $\tilde{\Omega}_{1}=a$ while $\Omega_{1}=1 \cdot k f_{1}=a g k / \omega \cosh k h$ [63], the dispersion relation is expressed as $\omega^{2}=g k \tanh k h$, so that the energy is:

$$
\begin{aligned}
\mathscr{E}_{1} & =\frac{1}{4}\left[a^{2}+\left(\frac{a g k}{\omega \cosh k h}\right)^{2} \cdot \frac{\sinh (2 k h)}{2 g k}\right] \\
& =\frac{1}{4}\left[a^{2}+\left(\frac{a^{2} g^{2} k^{2}}{g k \tanh k h \cdot \cosh ^{2} k h}\right) \cdot \frac{2 \sinh k h \cosh k h}{2 g k}\right]=\frac{a^{2}}{2},
\end{aligned}
$$




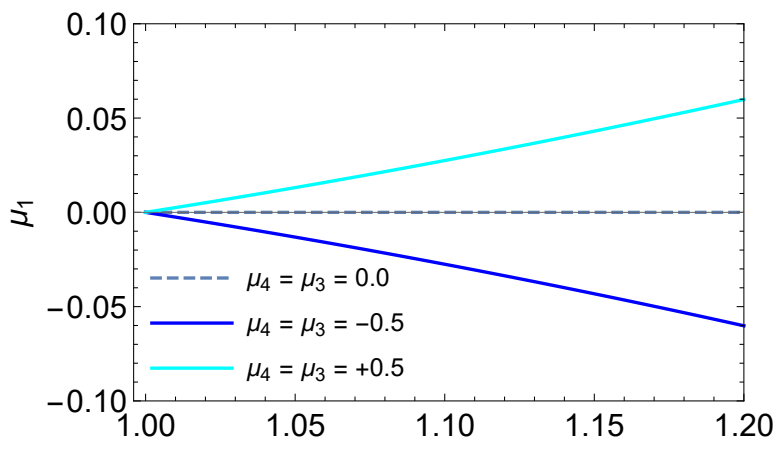

a)

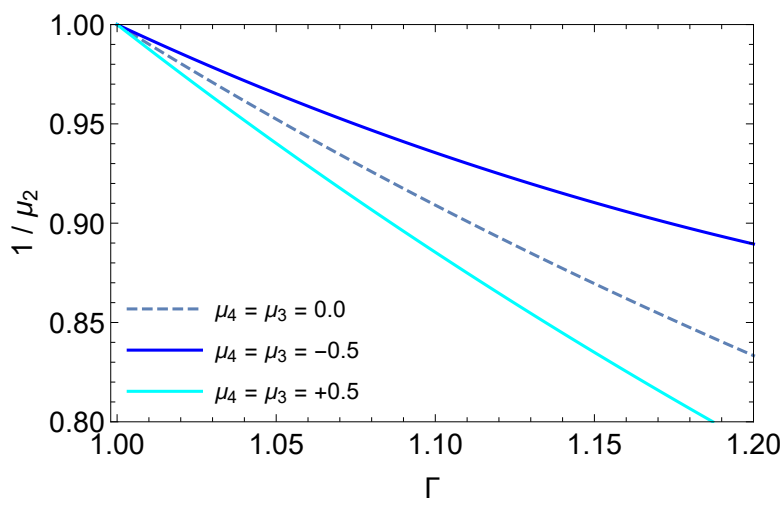

FIG. 6: $\Gamma$-GC model (a) ensemble average of the surface elevation and (b) its variance.

thus recovering the energy in eq. (6). For the second-order we have instead:

$$
\Omega_{1}=\frac{a \omega}{\sinh k h} ; \Omega_{2}=\frac{3 k a^{2} \omega}{4 \sinh ^{4} k h} ; \tilde{\Omega}_{1}=a ; \tilde{\Omega}_{2}=\frac{k a^{2} \cosh k h}{4 \sinh ^{3} k h}[2+\cosh (2 k h)] .
$$

Upon the steepness being expressed as $k a=(2 \pi / \lambda) \cdot(H / 2)=\pi \varepsilon$, the energy is computed and leads to eqs. (15)-(16):

$$
\begin{aligned}
\mathscr{E}_{2} & =\frac{a^{2}}{2}+\frac{1}{4}\left\{\frac{k^{2} a^{4}}{16}\left[\frac{\cosh k h}{\sinh ^{3} k h}(2+\cosh (2 k h))\right]^{2}+\left(\frac{3 k a^{2} \omega}{4 \sinh ^{4} k h}\right)^{2} \cdot \frac{\sinh (4 k h)}{4 g k}\right\}, \\
& =\frac{a^{2}}{4}\left\{2+\left(\frac{\pi \varepsilon}{4}\right)^{2}\left[\frac{\cosh k h}{\sinh ^{3} k h}(2+\cosh (2 k h))\right]^{2}+\left(\frac{\pi \varepsilon}{4}\right)^{2}\left[\frac{9 \cosh (2 k h)}{\sinh ^{6} k h}\right]\right\} .
\end{aligned}
$$

\section{Appendix C: Amplification Universality}

Here we shall prove the validity of the two relations in eq. (28). The result in eq. (28) assures us of the invariance of the ratio of logarithms of eq. (26) and of the amplification (ratio of probabilities) of eq. (27) regardless of the equilibrium exceedance probability prior to the shoal. Let us set up a general expression to accommodate both superRayleigh (+) and sub-Rayleigh (-) distributions, e.g. those who assign higher or lower probabilities than prescribed by Longuet-Higgins [12] either at the bulk or tail of the distribution. We attach a factor $g_{\mu \alpha}^{ \pm}$to the Rayleigh distribution, denoting a Gram-Charlier (hereby, abbreviated as GC) series [72, 98]. Eq. (28) holds if one can prove that the variance is corrected by a negligible term, denoted by $\mathfrak{L}_{\mu}$ :

$$
\mathbb{P}_{\alpha, \mu}^{ \pm}=g_{\mu \alpha}^{ \pm} \cdot \mathcal{R}_{\alpha} \quad \therefore \quad \mathbb{P}_{\alpha, \mu}^{ \pm}(\Gamma)=\left(\mathbb{P}_{\alpha, \mu}^{ \pm}\right)^{\frac{1}{\Gamma \pm \mathfrak{E}_{\mu}}}=\left(g_{\mu \alpha}^{ \pm} e^{-2 \alpha^{2}}\right)^{\frac{1}{\Gamma \pm \mathcal{E}_{\mu}}}
$$

We will show that this term $\mathfrak{L}_{\mu} \ll \Gamma$. Without loss of generality, the dual GC- $\zeta$ distribution with $\left|\mu_{3}\right|=\left|\mu_{4}\right|=1 / 2$ reads [72]:

$$
f_{\mu}^{ \pm}(\zeta) \equiv f_{\zeta}\left(m_{0}\right) \cdot g_{\mu \zeta}^{ \pm}=\frac{e^{-\zeta^{2} / 2 m_{0}}}{\sqrt{2 \pi m_{0}}} \cdot\left[1 \pm \frac{1}{12}\left(\zeta^{3}-3 \zeta\right) \pm \frac{1}{48}\left(\zeta^{4}-6 \zeta^{2}+3\right)\right]
$$

Clearly, the term $g_{\mu \alpha}^{ \pm}$is a by-product of the surface elevation counterpart $g_{\mu \zeta}^{ \pm}$. Hence, the following model captures the features of the two non-Gaussian distributions while being properly normalised:

$$
\frac{16}{16 \mp(\Gamma-1)^{2}} \int_{-\infty}^{+\infty} f_{\zeta}\left(m_{0} \Gamma\right) \cdot g_{\mu \zeta}^{ \pm} d \zeta=\int_{-\infty}^{+\infty} f_{\mu}^{ \pm}(\zeta, \Gamma) d \zeta=1
$$

With no loss of generality, let us set $m_{0}=1$ and the first moments read (see figure 6 ):

$$
\mu_{1}^{ \pm}=\int_{-\infty}^{+\infty} \frac{16 g_{\mu \zeta}^{ \pm} f_{\zeta}(\Gamma) \zeta}{16 \mp(\Gamma-1)^{2}} d \zeta= \pm \frac{4 \Gamma(\Gamma-1)}{\left[16 \mp(\Gamma-1)^{2}\right]},
$$




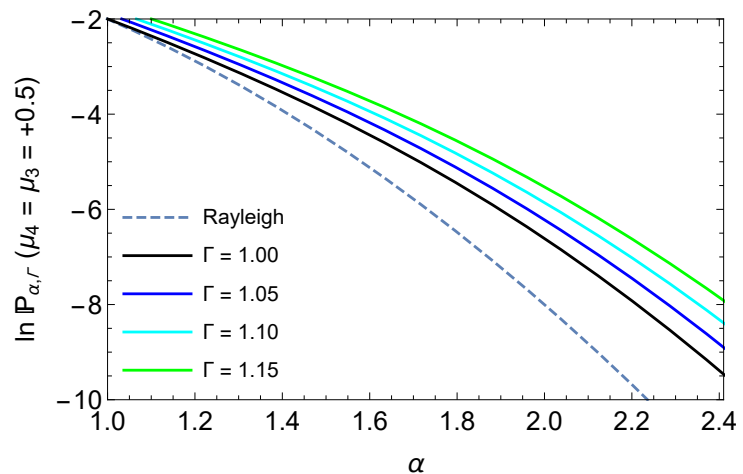

a)

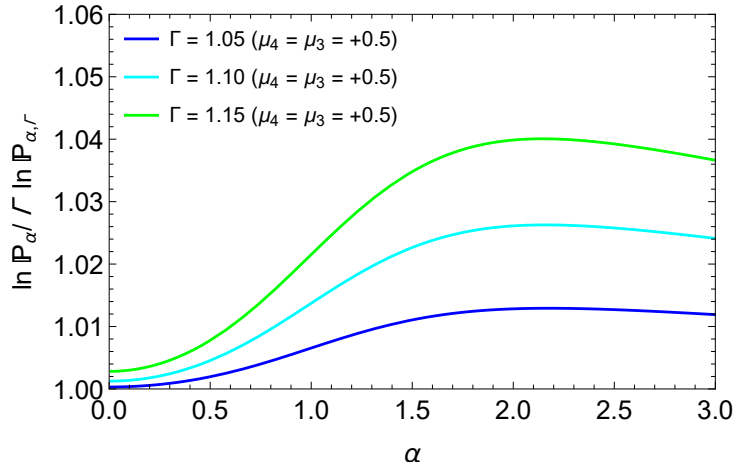

c)

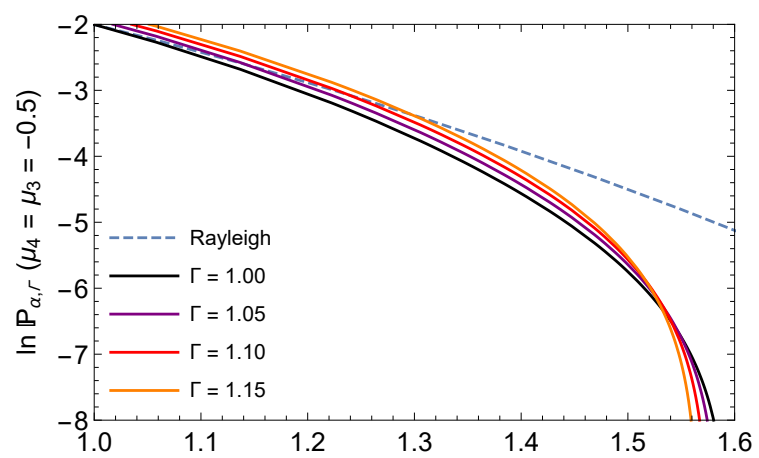

b)

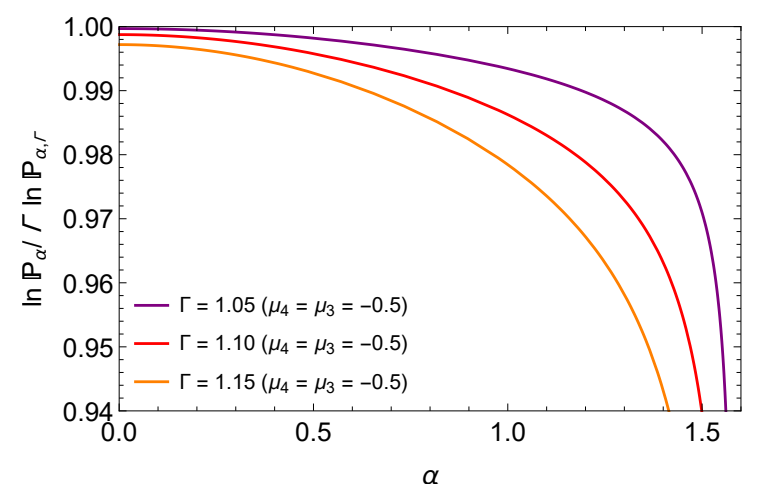

d) through $\mathfrak{L}_{\mu}$ in eq. (C10) is found for (c) super-Rayleigh and (d) sub-Rayleigh regimes.

while the sub-Rayleigh second moment is expressed as,

$$
\mu_{2}^{-}=\int_{-\infty}^{+\infty} \frac{16 g_{\mu \zeta}^{-} f_{\zeta}(\Gamma)\left(\zeta-\mu_{1}^{-}\right)^{2}}{16+(\Gamma-1)^{2}} d \zeta=\Gamma\left[1+\frac{\left(4 \Gamma^{4}-28 \Gamma^{3}-20 \Gamma^{2}+44 \Gamma\right)}{\left(15+2 \Gamma-\Gamma^{2}\right)^{2}}\right],
$$

and the super-Rayleigh as well (see figure 6),

$$
\mu_{2}^{+}=\int_{-\infty}^{+\infty} \frac{16 g_{\mu \zeta}^{+} f_{\zeta}(\Gamma)\left(\zeta-\mu_{1}^{+}\right)^{2}}{16-(\Gamma-1)^{2}} d \zeta=\Gamma\left[1+\frac{\left(4 \Gamma^{4}-28 \Gamma^{3}+108 \Gamma^{2}-84 \Gamma\right)}{\left(17-2 \Gamma+\Gamma^{2}\right)^{2}}\right]
$$

Comparing the variances with the exponent in eq. (C1), we find $\mu_{2}^{ \pm}=\Gamma\left(1 \pm \mathfrak{L}_{\mu} / \Gamma\right)$, and the corrections $\mathfrak{L}_{\mu}$ can be readily available by isolating the quotients inside the brackets of the r.h.s in the above equations. These coefficients can be further approximated as follows (recalling that $|\Gamma-1| \ll 1$ ):

$$
\left|\mu_{1}^{ \pm}\right| \approx \frac{(\Gamma-1)}{4} \quad ; \quad \mu_{2}^{ \pm} \approx \Gamma \pm \frac{(\Gamma-1)}{3} .
$$

Then, we showed a weak proof of the first part of eq. (28) by demonstrating that a change $g_{\mu \alpha}^{ \pm}$in the pre-shoal Rayleigh probability will always be met by a change $\pm \mathfrak{L}_{\mu}$ in the variance, typically obeying $\mathfrak{L}_{\mu} / \Gamma \ll 1$ (see figure $6 \mathrm{~b}$ ). We have also corroborated appendix A 2 on the negligibility of the $\mu_{1}$ amplification in comparison with the variance.

\section{Generalised Proof}

In this section we use the $\Gamma$-GC model to obtain exact closed-form wave height distributions, following the steps for the integration of the envelope in a two-dimensional random walk of Mori and Yasuda [72]. Thus we integrate 


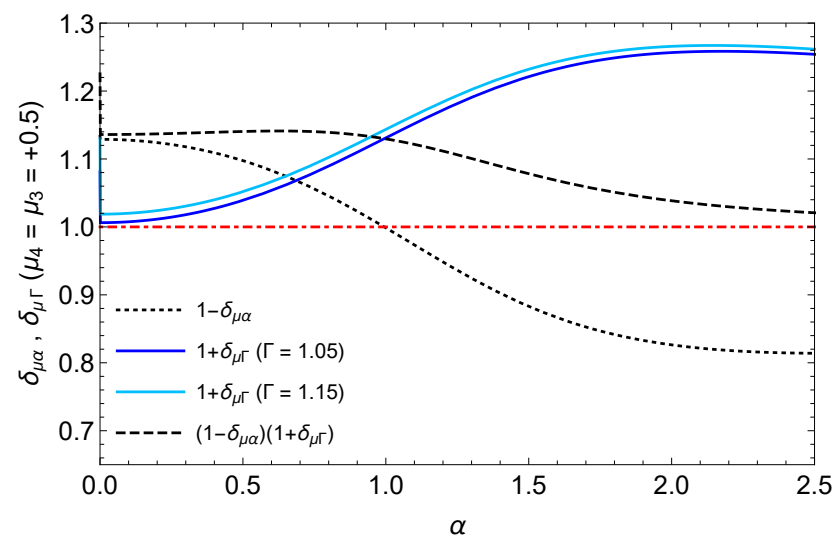

FIG. 8: Comparison between first-order corrections to the amplification of the $\Gamma$-GC exceedance probability for positive skewness and kurtosis as in eq. (C12).

the joint distribution of both surface elevation $\zeta$ and its Hilbert transform $\tilde{\zeta}$ over the uniform distribution of phases, obtaining the marginal density of the surface envelope:

$$
f_{\mu, R}^{ \pm}(\Gamma)=\int_{0}^{2 \pi} g_{\mu \zeta}^{ \pm} f_{\zeta}^{ \pm}(\Gamma) \cdot g_{\mu \tilde{\zeta}}^{ \pm} f_{\tilde{\zeta}}^{ \pm}(\Gamma) R d \phi
$$

where $\sqrt{\zeta^{2}+\tilde{\zeta}^{2}}=R$ is the envelope with $\zeta=R \cos \phi$ and $\tilde{\zeta}=R \sin \phi$. Performing this integration, changing variables to wave heights and later normalising by $H_{1 / 3}=4 m_{0}=4$ and integrating again as in eq. (2), we find (see figures $7 \mathrm{a}, \mathrm{b})$ :

$$
\begin{array}{r}
\mathbb{P}_{\alpha, \mu}^{ \pm}(\Gamma)=\frac{e^{-2 \alpha^{2} / \Gamma}}{3}\left[1+\frac{2 \alpha^{8}+4 \alpha^{6}(\Gamma-4)+6 \alpha^{4}\left(\Gamma^{2}-4 \Gamma+6 \pm 32\right)}{\left[16 \mp(\Gamma-1)^{2}\right]^{2}}\right. \\
\left.+\frac{3 \alpha^{2}(\Gamma-2)\left(\Gamma^{2}-2 \Gamma+2 \pm 32\right)}{\left[16 \mp(\Gamma-1)^{2}\right]^{2}}\right]
\end{array}
$$

Then, in analogy with eq. (26) we are able to assess whether or not the proposition in eq. (C1) holds by verifying the following ratio:

$$
\frac{\ln \left[\left(\mathbb{P}_{\alpha}^{ \pm}, \mu\right)\right]}{\left(\Gamma \pm \mathfrak{L}_{\mu}\right) \ln \left[\mathbb{P}_{\alpha, \mu}^{ \pm}(\Gamma)\right]}=1 \quad \therefore \quad \frac{\ln \left[\left(\mathbb{P}_{\alpha, \mu}^{ \pm}\right)\right]}{\Gamma \ln \left[\mathbb{P}_{\alpha, \mu}^{ \pm}(\Gamma)\right]}=1 \pm \frac{\mathfrak{L}_{\mu}}{\Gamma} \approx 1
$$

Accordingly, figures $7 \mathrm{c}, \mathrm{d}$ display the magnitude of the correction $1 \pm \mathfrak{L}_{\mu} / \Gamma$ in the variance. When $\Gamma \approx 1.15$ we see that super-Rayleigh distributions have a maximal $4 \%$ increase in the variance $\Gamma$, whereas sub-Rayleigh distributions exhibit the opposite but of smaller magnitude, confirming the estimates in the weak proof. As the validity of eq. (C1) has been demonstrated, one can prove the universality of the amplification regardless of the distribution, i.e. extend eq. (27) to an arbitrary distribution. Having in mind the order of magnitude of $\mathfrak{L}_{\mu} / \Gamma$, we can rewrite eq. (C1) as follows (defining $\left|\ln g_{\mu \alpha}^{ \pm}\right|=\ln g_{\mu \alpha}$ ):

$$
\mathbb{P}_{\alpha, \mu}^{ \pm}(\Gamma)=\left(e^{-2 \alpha^{2} \pm \ln g_{\mu \alpha}}\right)^{\frac{1}{\Gamma \pm \mathfrak{s} \mu}} \approx \exp \left[-\frac{2 \alpha^{2}}{\Gamma}\left(1 \mp \frac{\ln g_{\mu \alpha}}{2 \alpha^{2}}\right)\left(1 \mp \frac{\mathfrak{L}_{\mu}}{\Gamma}\right)\right]
$$


Furthermore, the relative probability becomes:

$$
\begin{aligned}
\frac{\mathbb{P}_{\alpha, \mu}^{ \pm}(\Gamma)}{\mathbb{P}_{\alpha, \mu}^{ \pm}} & =\exp \left[\left(-2 \alpha^{2} \pm \ln g_{\mu \alpha}\right)\left(\frac{1}{\Gamma \pm \mathfrak{L}_{\mu}}-1\right)\right], \\
& =\exp \left[2 \alpha^{2}\left(1 \mp \frac{\ln g_{\mu \alpha}}{2 \alpha^{2}}\right)\left(1-\frac{1}{\Gamma \pm \mathfrak{L}_{\mu}}\right)\right], \\
& =\exp \left[2 \alpha^{2}\left(1-\frac{1}{\Gamma}\right)\left(1 \mp \frac{\ln g_{\mu \alpha}}{2 \alpha^{2}}\right)\left(1 \pm \frac{\mathfrak{L}_{\mu}}{\Gamma^{2}\left(1-\frac{1}{\Gamma}\right)}\right)\right], \\
& =\exp \left[2 \alpha^{2}\left(1-\frac{1}{\Gamma}\right)\left(1 \mp \frac{\ln g_{\mu \alpha}}{2 \alpha^{2}}\right)\left(1 \pm \frac{\mathfrak{L}_{\mu}}{\Gamma(\Gamma-1)}\right)\right], \\
& \equiv \exp \left[2 \alpha^{2}\left(1-\frac{1}{\Gamma}\right)\left(1 \mp \delta_{\mu \alpha}\right)\left(1 \pm \delta_{\mu \Gamma}\right)\right] .
\end{aligned}
$$

As $|\Gamma-1| \ll 1$, it is straightforward to see that $\delta_{\mu \Gamma}=\mathfrak{L}_{\mu} / \Gamma(\Gamma-1) \sim 10 \mathfrak{L}_{\mu} \sim \delta_{\mu \alpha}=\left(\ln g_{\mu \alpha}\right) / 2 \alpha^{2}$, plotted for comparison in figure 8. Hence, we conclude that for the probability amplification the second correction term $\delta_{\mu \Gamma}$ counters $\delta_{\mu \alpha}$. Thus, we have proved the first-order amplification universality with $\mu_{3} \sim \mu_{4}<1$ :

$$
\frac{\mathbb{P}_{\alpha, \mu}^{ \pm}(\Gamma)}{\mathbb{P}_{\alpha, \mu}^{ \pm}}=e^{2 \alpha^{2}\left(1-\frac{1}{\Gamma}\right) \pm \mathcal{O}(\Gamma-1)} \approx e^{2 \alpha^{2}\left(1-\frac{1}{\Gamma}\right)} \equiv \frac{\mathcal{R}_{\alpha}(\Gamma)}{\mathcal{R}_{\alpha}} \quad, \quad \forall g_{\mu \alpha}^{ \pm} \in \mathbb{R}_{+}
$$

Rogue waves in eq. (29) have $2 \alpha^{2}\left[1-\left(\mathfrak{S}_{0}^{2} \Gamma\right)^{-1}\right] \leqslant 3$, resulting in maximal correction $e^{\mathcal{O}(\Gamma-1)} \approx e^{3 \times 0.03}<1.1$, suggesting an upper bound of 8-9\% variation from the universal amplification. On the other hand, figure 8 shows $\left(1 \mp \delta_{\mu \alpha}\right)\left(1 \pm \delta_{\mu \Gamma}\right) \approx 1.14$ for ordinary waves $(\alpha \leqslant 1)$ instead of $\left(1 \mp \delta_{\mu \alpha}\right)\left(1 \pm \delta_{\mu \Gamma}\right) \leqslant 1.04$ for rogue waves, such that the main term of eq. $(29)$ becomes $2 \alpha^{2}\left[1-\left(\mathfrak{S}_{0}^{2} \Gamma\right)^{-1}\right] \leqslant 1$, and the first-order correction reads $e^{\mathcal{O}(\Gamma-1)} \approx e^{0.6 \times 0.15}<1.1$. Hence, the bound is upheld by any normalised wave height.

\section{Appendix D: Parameterisation generality and compact formulation}

Under adequate conditions of Ur $\leqslant 8 \pi^{2} / 3$ [49] and for shoals in Regime II, eq. (31) can be rewritten in a more compact form. First, however, let us demonstrate that eq. (31) holds regardless of which reference steepness is chosen to compute $\kappa_{0}$. For relatively higher or lower reference steepness $\varepsilon_{ \pm}=\langle\varepsilon\rangle \pm \delta \varepsilon$, we find:

$$
\kappa_{0}^{ \pm}=\frac{\ln \left[\max \mathfrak{S}_{0}\right]}{\ln \left[\max \Gamma\left(\langle\varepsilon\rangle \pm \delta \varepsilon, k_{p} h, \mathfrak{S}_{0}\right)\right]} \approx \kappa_{0} \mp \delta \kappa_{0}
$$

in turn affecting the probability amplification only marginally:

$$
\left(\frac{\ln \mathbb{P}_{\alpha}}{\ln \mathbb{P}_{\alpha, \Gamma}}\right)_{ \pm}=\left[\Gamma_{0} \pm \delta \Gamma_{0}\right]^{2 \kappa_{0} \mp 2 \delta \kappa_{0}} \cdot \Gamma\left(\varepsilon(x), k_{p} h(x), \mathfrak{S}_{0}\right) \approx \frac{\ln \mathbb{P}_{\alpha}}{\ln \mathbb{P}_{\alpha, \Gamma}}
$$

The above relation holds because the typical difference between the $\Gamma$ correction atop the shoal and the average moving during the shoal does not exceed $\delta \Gamma_{0} / \Gamma_{0} \leqslant 2 \%$. We corroborate this by comparing the equivalent of figure 5 (panel a) for a $0.5 \%$ higher $\Gamma$ correction than its reference $\Gamma_{0}=1.031$ in figure $9 \mathrm{a}$. Considering that Run 1 had maximal correction $\Gamma \approx 1.041$, the choice for the reference $\kappa_{0}$ does not affect the validity of eq. (31). To obtain a compact formulation of eq. (31), we notice that at a fixed point in space, i.e. at a distance $x=x_{i}$ from the wavemaker, the asymmetry $\mathfrak{S}_{0}$ does not depend on how we plot $\Gamma$. Therefore, we can approximate

$$
\mathfrak{S}_{0}\left(x=x_{i}\right)=\left[\Gamma\left(\varepsilon\left(x_{i}\right), k_{p} h\left(x_{i}\right), \mathfrak{S}_{0}\right)\right]^{\kappa_{x}} \approx\left[\Gamma\left(\langle\varepsilon\rangle, k_{p} h, \mathfrak{S}_{0}\right)\right]^{\kappa_{0}}, \forall x_{i} \in \mathbb{R} .
$$

As the two versions of the $\Gamma$ correction differ, as shown in figure $9 \mathrm{~b}$, one concludes that $\kappa\left(k_{p} h\right) \equiv \kappa_{0} \neq \kappa_{x}$. Thus we obtain:

$$
\ln \mathfrak{S}_{0} \approx\left\langle\kappa_{x}\right\rangle \ln \langle\Gamma(x)\rangle \approx \kappa_{0} \ln \left\langle\Gamma\left(\langle\varepsilon\rangle, k_{p} h, \mathfrak{S}_{0}\right)\right\rangle
$$

whose Regime II restriction is numerically translated to $\Gamma \geqslant 1+\mathcal{O}\left(g^{-2}\right)$, leading to $\left\langle\kappa_{x}\right\rangle \approx 0.64 \kappa_{k h}$, as shown in figure 9b. Therefore, we can conservatively estimate throughout the entire trajectory: 

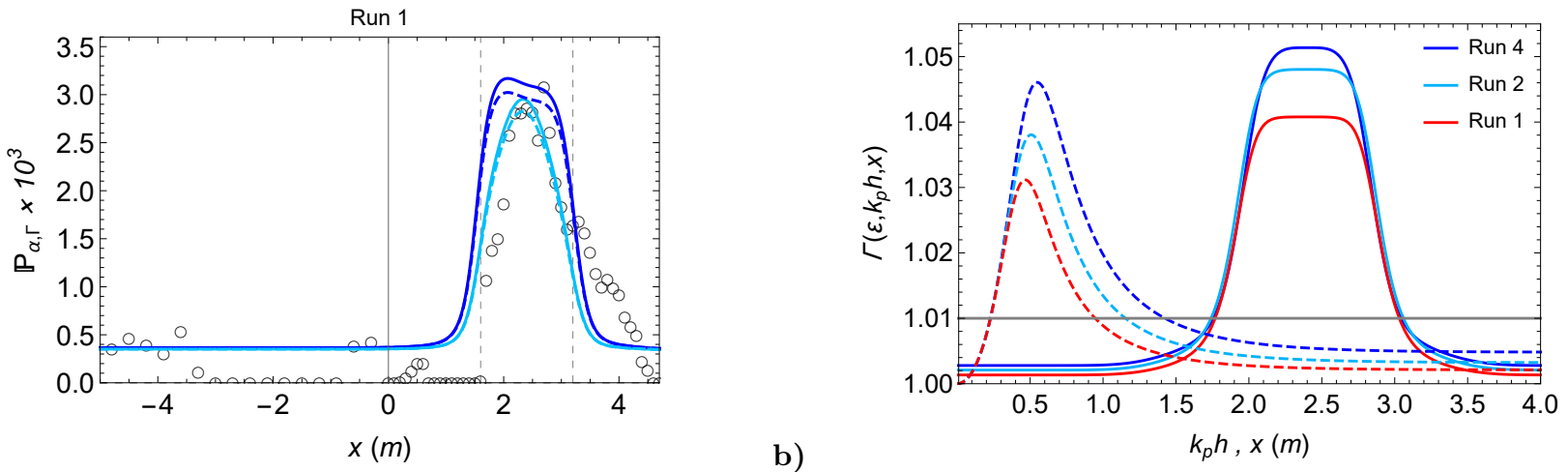

a)

b)

FIG. 9: (a) Equivalent of figure 5a for a higher reference $\Gamma$ correction due to steepness $\varepsilon_{+} \approx 1.16\langle\varepsilon\rangle$ (dashed) and its original description according to eq. (31) (solid). Notice that this $16 \%$ increase in the reference steepness decreases the blue curve model by $4 \%$ and the cyan one by $2 \%$. (b) Plot of $\Gamma$ correction for runs 1-4 of Raustøl [26] as a function of dimensionless depth (dashed) and distance from the wavemaker (solid) with variables $(\varepsilon, k h)$ modelled by appendix E, whereas the minimum threshold applicable $(\Gamma \geqslant 1.01)$ representative of Regime II is depicted by the thin horizontal line. The averages over these ranges approximately read $\langle\Gamma(x)\rangle=1.036$ and $\left\langle\Gamma\left(k_{p} h\right)\right\rangle=1.023$.

\begin{tabular}{l|r|rrrrrrrrrrrrrr}
\hline \multicolumn{1}{c|}{$\operatorname{Exp}}$. & $\varepsilon_{1}$ & $\varepsilon_{2}$ & $\varepsilon_{3}$ & $\varepsilon_{4}$ & $\delta_{31}$ & $\delta_{32}$ & $\delta_{41}$ & $\delta_{42}$ & $D_{1}$ & $D_{2}$ & $D_{3}$ & $D_{4}$ & $\delta_{62}$ & $\delta_{82}$ \\
\hline Run 1 & 0.0230 & 0.0230 & 0.0030 & -0.0070 & - & - & - & - & 1.85 & -1.32 & -0.40 & -0.50 & - & - \\
Run 2 & 0.0306 & 0.0242 & 0.0020 & -0.0050 & - & - & - & - & 2.08 & -1.50 & -0.45 & -0.45 & - & 0.1 \\
Run & 4 & 0.0380 & 0.0240 & 0.0020 & -0.0060 & - & - & - & - & 2.60 & -1.93 & -0.60 & -0.60 & - & 0.1 \\
Run 5 & 0.0460 & 0.0200 & 0.0020 & -0.0080 & - & - & - & 0.3 & 3.20 & -2.40 & -0.80 & -0.75 & - & 0.1 \\
Run 6 & 0.0600 & 0.0167 & -0.0020 & -0.0100 & - & - & -0.5 & 0.4 & 4.15 & -3.20 & -0.99 & -0.99 & -0.1 & 0.1 \\
Run 7 & 0.0610 & 0.0150 & 0.0020 & -0.0030 & - & - & - & - & 2.50 & -1.50 & -0.50 & -0.50 & -0.1 & 0.1 \\
Run 8 & 0.0695 & 0.0105 & 0.0015 & -0.0040 & - & - & - & 0.1 & 3.05 & -2.00 & -0.80 & -0.80 & - & 0.1 \\
Run 9 & 0.0672 & 0.0073 & 0.0005 & -0.0040 & - & - & - & 0.5 & 3.40 & -2.15 & -0.55 & -0.60 & -0.1 & 0.1 \\
Run 11 & 0.0755 & 0.0015 & -0.0008 & -0.0030 & - & 0.4 & -0.5 & 0.7 & 4.30 & -2.85 & -0.85 & -0.80 & -0.1 & 0.1 \\
Run 12 & 0.0760 & 0.0002 & -0.0025 & -0.0072 & -0.4 & -0.7 & 0.5 & 4.95 & -3.30 & -1.00 & -1.00 & -0.1 & 0.1 \\
\hline
\end{tabular}

TABLE I: Summary of all coefficients for the modelling in eqs. (E1-E2).
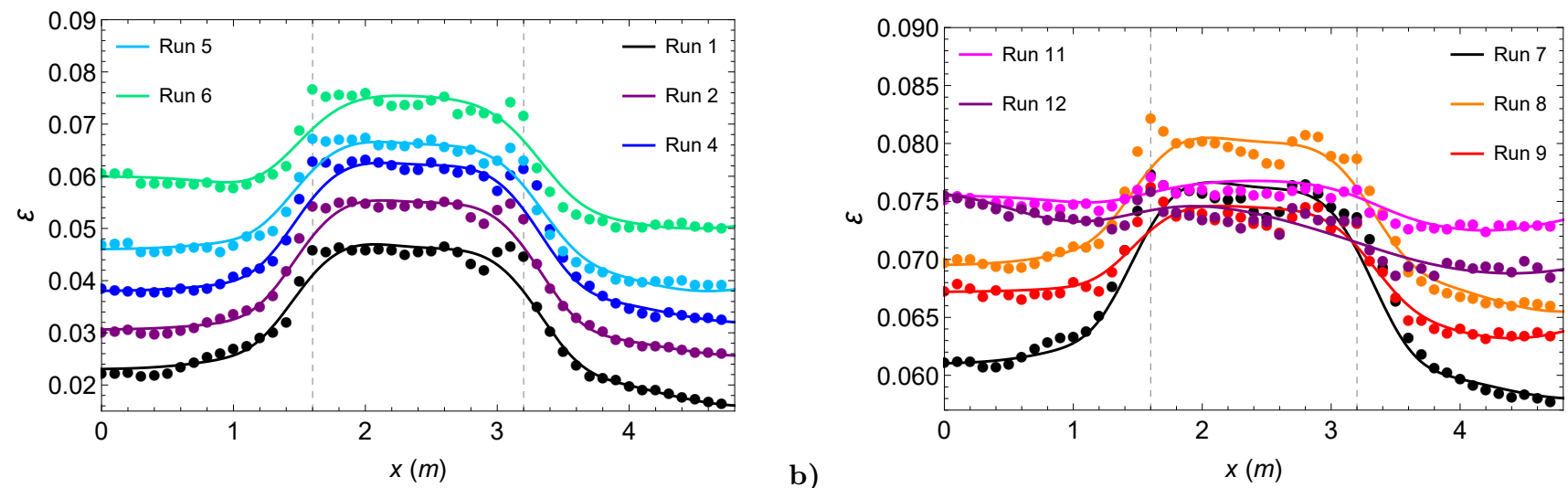

FIG. 10: Modelling of the significant steepness in Raustøl [26] experiments according to eq. (E1), corrected to the term $\varepsilon=H_{1 / 3} / \bar{\lambda}=\frac{4}{\pi} k_{\mathrm{p}} a_{c}$. Dots represent data extracted from Figure 5.4 of Raustøl [26]. Vertical dashed lines depict the rising shoal end $(x=1.6)$ and beginning of the descending shoal $(x=3.2)$. 


$$
\left[\Gamma\left(\langle\varepsilon\rangle, k_{p} h, \mathfrak{S}_{0}\right)\right]^{2 \kappa_{0}} \approx\left[\Gamma\left(\varepsilon(x), k_{p} h(x), \mathfrak{S}_{0}\right)\right]^{1.2 \kappa_{0}}
$$

Thus, the ratio of the probabilities is better estimated and greatly simplified as:

$$
\frac{\ln \mathbb{P}_{\alpha}}{\ln \mathbb{P}_{\alpha, \Gamma}} \approx\left[\Gamma\left(\varepsilon(x), k_{p} h(x), \mathfrak{S}_{0}\right)\right]^{1+1.2 \kappa_{0}}
$$

\section{Appendix E: Analytical Description of Steepness and Depth}

In order to smooth as well as to facilitate the handling of the experimental data on wave steepness and dimensionless depth, we fitted them against analytic functions, as follows:

$$
\varepsilon=\varepsilon_{1}+\varepsilon_{2} \mathrm{e}^{-(x-2.4)^{4}}+\varepsilon_{3} \mathrm{e}^{-\left(2+\delta_{31}\right)\left[x-1.4+\delta_{32}\right]^{2}}+\varepsilon_{4} \mathrm{e}^{-\left(1+\delta_{41}\right)\left[x-4.9+\delta_{42}\right]^{2}},
$$

while the modelling for the depth is computed as,

$$
k_{\mathrm{p}} h=D_{1}+D_{2} \mathrm{e}^{-0.25(x-2.4)^{4}}+D_{3} \mathrm{e}^{-2\left[x+\delta_{62}-0.7\right]^{2}}+D_{4} \mathrm{e}^{-2\left[x+\delta_{82}-4.2\right]^{2}} .
$$

The values of these coefficients for ten runs of Raustøl [26], Trulsen et al. [31] are given in table I. As displayed in figure 10, these fits provide an accurate description of the actual data. Re-scaled by $\pi / 4$, the first steepness coefficient $\varepsilon_{1}$ is equal to the pre-shoal steepness in table 1 of Trulsen et al. [31] while $\varepsilon_{1}+\varepsilon_{2}$ is the shallower steepness of the shoal. Likewise, the coefficient $D_{1}$, which was extracted from Raustøl [26] through the formula $k_{\mathrm{p}} h \approx(\pi \varepsilon / 4 \mathrm{Ur})^{1 / 3}$ (see Trulsen et al. [31]), approximately equals the largest values of the dimensionless depth, while $D_{1}+D_{2}$ recovers the smallest values. Note, however, that Trulsen et al. [31] displays the averages of each sides, while we model every value according to the 16 probes of figure 2 in Trulsen et al. [31].

[1] A. Toffoli, J. Lefevre, E. Bitner-Gregersen, and J. Monbaliu, Towards the identification of warning criteria: Analysis of a ship accident database, Appl. Ocean Res. 27, 281 (2005).

[2] K. Dysthe, H. Krogstad, and P. Muller, Oceanic rogue waves, Annu. Rev. Fluid. Mech. 40, 287 (2008).

[3] M. Onorato, S. Residori, U. Bortolozzo, A. Montina, and F. Arecchi, Rogue waves and their generating mechanisms in different physical contexts, Phys. Rep. 528, 47 (2013).

[4] S. Haver, Evidences of the existence of freak waves, In: M. Olagnon and G.A. Athanassoulis (Eds.), Rogue Waves 2000 , 129 (2000).

[5] D. Faulkner and W. Buckley, Critical survival conditions for ship design, International Conference on Design and Operation for Abnormal Conditions, RINA 6, 1 (1997).

[6] D. Faukner, Shipping safety: A matter of concern, Ingenia, The Royal Academy of Engineering, Marine Matters , 13 (2002).

[7] T. B. Benjamin and J. E. Feir, The disintegration of wave trains on deep water part 1. theory, Journal of Fluid Mechanics 27, 417 (1967).

[8] V. Zakharov and L. Ostrovsky, Modulation instability: The beginning, Physica D: Nonlinear Phenomena 238, 540 (2009).

[9] P. Boccotti, Wave mechanics for ocean engineering, Elsevier Oceanography Series (2000).

[10] F. Fedele, J. Brennan, S. De Leon, J. Dudley, and F. Dias, Real world ocean rogue waves explained without the modulational instability, Sci. Rep. 6, 27715 (2016).

[11] G. Dematteis, T. Grafke, M. Onorato, and E. Vanden-Eijnden, Experimental evidence of hydrodynamic instantons: The universal route to rogue waves, Phys. Rev. X 91 (2019).

[12] M. Longuet-Higgins, On the statistical distribution of the heights of sea waves, Journal of Marine Research 11, 245 (1952).

[13] S. Rice, Mathematical analysis of random noise, Bell Syst. Tech. J. 24, 46 (1945).

[14] M. St Denis and W. J. Pierson, On the motions of ships in confused seas, Trans. Soc. Nav. Archit., N.Y. 61, 1 (1953).

[15] P. Stansell, Distribution of freak wave heights measured in the north sea, Appl. Ocean Res. 26, 35 (2004).

[16] B. Glukhovskii, Investigation of sea wind waves (in russian), Gidrometeoizdat (1966).

[17] I. Karmpadakis, C. Swan, and M. Christou, Assessment of wave height distributions using an extensive field database, Coastal Eng. 157 (2020).

[18] S. Mendes, A. Scotti, and P. Stansell, On the physical constraints for the exceeding probability of deep water rogue waves, Appl. Ocean Res. 108, 102402 (2021).

[19] O. Phillips, The equilibrium range in the spectrum of wind-generated waves, Journal of Fluid Mechanics 4, 426 (1958).

[20] K. Hasselmann, On the non-linear energy transfer in a gravity-wave spectrum: Part 1. general theory, Journal of Fluid Mechanics 12, 481 (1962). 
[21] W. J. Pierson and L. Moskowitz, A proposed spectral form for fully developed wind seas based on the similarity theory of s. a. kitaigorodskii, J. Geophys. Res. 69, 5181 (1964).

[22] M. Donelan, W. Drennan, and A. Magnusson, Nonstationary analysis of the directional properties of propagating waves, Journal of Physical Oceanography 26, 1901 (1996).

[23] S. Haver and O. Andersen, Freak waves: Rare realizations of a typical population of typical realizations of a rare population?, Proc. 10th Int. Offshore Polar Eng. Conf. Seattle 3, 123 (2000).

[24] K. Trulsen, H. Zeng, and O. Gramstad, Laboratory evidence of freak waves provoked by non-uniform bathymetry, Phys. Fluids 24 (2012).

[25] K. Trulsen, Rogue waves in the ocean, the role of modulational instability, and abrupt changes of environmental conditions that can provoke non equilibrium wave dynamics, in: The Ocean in Motion: Circulation, Waves, Polar Oceanography (2018).

[26] A. Raustøl, Freake bølger over variabelt dyp, Master's thesis, University of Oslo (2014).

[27] Y.-X. Ma, X.-Z. Ma, and G.-H. Dong, Variations of statistics for random waves propagating over a bar, Journal of Marine Science and Technology (Taiwan) 23, 864 (2015).

[28] C. Bolles, K. Speer, and M. Moore, Anomalous wave statistics induced by abrupt depth change, Physical Review Fluids 4 (2019).

[29] J. Zhang, M. Benoit, O. Kimmoun, A. Chabchoub, and H.-C. Hsu, Statistics of extreme waves in coastal waters: Large scale experiments and advanced numerical simulations, Fluids 4 (2019).

[30] L. Zou, A. Wang, Z. Wang, Y. Pei, and X. Liu, Experimental study of freak waves due to three-dimensional island terrain in random wave, Acta Oceanologica Sinica 38, 92 (2019).

[31] K. Trulsen, A. Raust $\varnothing$ l, S. Jorde, and L. Rye, Extreme wave statistics of long-crested irregular waves over a shoal, J. Fluid Mech. 882 (2020).

[32] H. Zeng and K. Trulsen, Evolution of skewness and kurtosis of weakly nonlinear unidirectional waves over a sloping bottom, Nat. Hazards Earth Syst. Sci. 12, 631 (2012).

[33] O. Gramstad, H. Zeng, K. Trulsen, and G. Pedersen, Freak waves in weakly nonlinear unidirectional wave trains over a sloping bottom in shallow water, Physics of Fluids 25 (2013).

[34] G. Ducrozet and M. Gouin, Influence of varying bathymetry in rogue wave occurrence within unidirectional and directional sea-states, Journal of Ocean Engineering and Marine Energy 3 (2017).

[35] Y. Zheng, Z. Lin, Y. Li, T. Adcock, Y. Li, and T. Van Den Bremer, Fully nonlinear simulations of unidirectional extreme waves provoked by strong depth transitions: The effect of slope, Phys. Rev. Fluids 5 (2020).

[36] J. Zhang and M. Benoit, Wave-bottom interaction and extreme wave statistics due to shoaling and de-shoaling of irregular long-crested wave trains over steep seabed changes, Journal of Fluid Mechanics 912, A28 (2021).

[37] A. Majda, M. Moore, and D. Qi, Statistical dynamical model to predict extreme events and anomalous features in shallow water waves with abrupt depth change, Proceedings of the National Academy of Sciences of the United States of America 116, 3982 (2019).

[38] N. Moore, C. Bolles, A. Majda, and D. Qi, Anomalous waves triggered by abrupt depth changes: Laboratory experiments and truncated kdv statistical mechanics, Journal of Nonlinear Science 30, 3235 (2020).

[39] Y. Li, S. Draycott, T. A. Adcock, and T. Van Den Bremer, Surface wavepackets subject to an abrupt depth change. part 2: Experimental analysis, J. Fluid Mech. 915, A72 (2021).

[40] Y. Li, Y. Zheng, Z. Lin, T. A. Adcock, and T. Van Den Bremer, Surface wavepackets subject to an abrupt depth change. part 1: Second-order theory, J. Fluid Mech. 915, A71 (2021).

[41] Y. Li, S. Draycott, Y. Zheng, Z. Lin, T. Adcock, and T. Van Den Bremer, Why rogue waves occur atop abrupt depth transitions, Journal of Fluid Mechanics 919, R5 (2021).

[42] M. A. Tayfun, Narrow-band nonlinear sea waves, J. Geophys. Res. 85, 1548 (1980).

[43] S. Massel, Ocean surface waves: Their physics and prediction, 3rd ed. (World Scientific, Singapore, 2017).

[44] S. Kjeldsen, Dangerous wave groups, Nor. Marit. Res. 12, 4 (1984).

[45] D. Myrhaug and S. Kjeldsen, Steepness and asymmetry of extreme waves and the highest waves in deep water, Ocean Engineering 13, 549 (1986).

[46] B. Linfoot, P. Stansell, and J. Wolfram, On the characteristics of storm waves, Proceedings of the International Offshore and Polar Engineering Conference 3, 74 (2000).

[47] C. Guedes Soares, Z. Cherneva, and E. Antao, Steepness and asymmetry of the largest waves in storm sea states, Ocean Engineering 31, 1147 (2004).

[48] M. Tayfun, Statistics of nonlinear wave crests and groups, Ocean Eng. 33, 1589 (2006).

[49] R. Dean and R. Dalrymple, Water wave mechanics for engineers and scientists., World Scientific (1984).

[50] G. B. Airy, Tides and waves, in encyclopaedia metropolitana, B.Fellowes, London 122, 241-396 (1845).

[51] A. Khintchine, Korrelationstheorie der stationären stochastischen prozesse, Math. Ann. 109, 604 (1934).

[52] Z. Cherneva and C. Guedes Soares, Non-linearity and non-stationarity of the new year abnormal wave, Appl. Ocean Res. 30, 215 (2008).

[53] Y. Goda, Random seas for design of maritime structures, World Scientific (2010).

[54] M. S. Longuet-Higgins, On the distribution of the heights of sea waves: Some effects of nonlinearity and finite band width, J. Geophys. Res. 85, 1519 (1980).

[55] S. Das and G. Nason, Measuring the degree of non-stationarity of a time series, Stat 5, 295 (2016).

[56] M. A. Tayfun, Distributions of envelope and phase in wind waves, J. Phys. Oceanogr. 38, 2784 (2008). 
[57] R. M. Loynes, On the concept of the spectrum for non-stationary processes, Journal of the Royal Statistical Society. Series B (Methodological) 30, 1 (1968).

[58] L. Cohen, Time-frequency distributions - a review, Proceedings of the IEEE 77, 941 (1989).

[59] P. Flandrin, Time-dependent spectra for non-stationary stochastic processes, in Time and Frequency Representation of Signals and Systems (Springer Vienna, Vienna, 1989) pp. 69-124.

[60] S. Adak, Time-Dependent Spectral Analysis of Nonstationary Time Series (Stanford University, 1995).

[61] A. Bruscato and C. M. Toloi, Spectral analysis of non-stationary processes using the fourier transform, Brazilian Journal of Probability and Statistics , 69 (2004).

[62] P. Janssen, On a random time series analysis valid for arbitrary spectral shape, J. Fluid Mech. 759, 236 (2014).

[63] M. W. Dingemans, Water Wave Propagation Over Uneven Bottoms (World Scientific, 1997).

[64] G. Forristall, Wave crest distributions: observations and second order theory, J. Phys. Ocean. 30, 1931 (2000).

[65] P. S. Eagleson, Properties of shoaling waves by theory and experiment, Eos, Transactions American Geophysical Union 37, 565 (1956).

[66] R. Fu, Y. Ma, G. Dong, and M. Perlin, A wavelet-based wave group detector and predictor of extreme events over unidirectional sloping bathymetry, Ocean Eng. 229 (2021).

[67] B. Lé Méhaute, An introduction to hydrodynamics and water waves, Springer (1976).

[68] R. Miche, Mouvements ondulatoires de la mer en profendeur constante ou decroissante forme limite de la houle lors de son deferlement, Ann. Ponts Chaussees 121, 285-319 (1944).

[69] Y. Goda, A unified nonlinearity parameter of water waves, Rept. Port and Harbour Res. Inst. 22 (3), 3 (1983).

[70] J. Vandever, E. Siegel, J. Brubaker, and C. Friedrichs, Influence of spectral width on wave height parameter estimates in coastal environments, Journal of Waterway, Port, Coastal and Ocean Engineering 134, 187 (2008).

[71] C. Tung and N. Huang, Peak and trough distributions of nonlinear waves, Ocean Eng. 12, 201 (1985).

[72] N. Mori and T. Yasuda, A weakly non-gaussian model of wave height distribution random wave train, Ocean Eng. 29, 1219-1231 (2002).

[73] M. Tayfun, Distribution of large wave heights, J. Waterway, Port, Coastal Ocean Eng. 116, 686 (1990).

[74] M. A. Tayfun and F. Fedele, Wave-height distributions and nonlinear effects, Ocean Eng. 34, 1631 (2007).

[75] A. Naess, On the distribution of crest to trough wave heights, Ocean Eng. 12, 221 (1985).

[76] M. A. Alkhalidi and M. A. Tayfun, Generalized boccotti distribution for nonlinear wave heights, Ocean Eng. 74, 101 (2013).

[77] T. Marthinsen, On the statistics of irregular second-order waves, Report No. RMS-11 (1992).

[78] M. A. Tayfun and M. A. Alkhalidi, Distribution of sea-surface elevations in intermediate and shallow water depths, Coastal Eng. 157 (2020).

[79] S. Jorde, Kinematiken i bølger over en grunne, Master's thesis, University of Oslo (2018).

[80] F. Barbariol, A. Benetazzo, S. Carniel, and M. Sclavo, Space-time wave extremes: The role of metocean forcings, J. Phys. Oceanogr. 45, 1897 (2015).

[81] M. Onorato and P. Suret, Twenty years of progresses in oceanic rogue waves: the role played by weakly nonlinear models, Natural Hazards 84, 541 (2016).

[82] M. Christou and K. Ewans, Field measurements of rogue water waves, J. Phys. Oceanogr. 9, 2317 (2014).

[83] A. Cattrell, M. Srokosz, B. Moat, and R. Marsh, Can rogue waves be predicted using characteristic wave parameters?, J. Geophys. Res. Oceans 123, 5624 (2018).

[84] B. D. Ripley, Spatial Statistics (Wiley-Interscience, 1981) p. 260pp.

[85] M. Sherman, Spatial Statistics and Spatio-Temporal Data: Covariance Functions and Directional Properties (Wiley series on applied probability, 2010) p. 297pp.

[86] M. Shinozuka and C.-M. Jan, Digital simulation of random processes and its applications, Journal of Sound and Vibration 25, 111 (1972).

[87] M. Shinozuka and G. Deodatis, Stochastic process models for earthquake ground motion, Probabilistic Engineering Mechanics 3, 114 (1988).

[88] G. Deodatis and M. Shinozuka, Digital simulation of seismic ground motion using stochastic wave theory, Proceedings 9th World Conference on Earthquake Engineering 2 (1988).

[89] L. Boltzmann, Vorlesungen für Gastheorie, II. Teil (J. A. Barth, Leipzig, 1898).

[90] O. Penrose, Foundations of Statistical Mechanics (Oxford University Press, 1973).

[91] H. Cramér and M. R. Leadbetter, Stationary and related stochastic processes, J. Wiley \& Sons, New York (1967).

[92] W. Davenport and W. Root, An introduction to the theory of random signals and noise, Wiley-IEEE Press (1987).

[93] D. Middleton, An introduction to statistical communication theory, Wiley-IEEE (1996).

[94] Y. Choi, Y. V. Lvov, and S. Nazarenko, Probability densities and preservation of randomness in wave turbulence, Physics Letters A 332, 230 (2004).

[95] Y. Choi, Y. V. Lvov, and S. Nazarenko, Joint statistics of amplitudes and phases in wave turbulence, Physica D 201, 121 (2005).

[96] A. Papoulis, Probability, Random Variables and Stochastic Processes (McGraw-Hill, 4th ed., New York, 2002).

[97] J. Blitzstein and J. Hwang, Introduction to probability (CRC Press, Taylor \& Francis Group, 2nd ed., 2019).

[98] M. Longuet-Higgins, The effect of non-linearities on statistical distributions in the theory of sea waves, J. Fluid Mech. 17, 459 (1963). 